Revue des patrimoines

\title{
Chargé de la protection des monuments historiques : un professionnel du patrimoine au métier diversifié
}

Designation of historic monuments, a heritage profession calling on multiple skills

pour l'association MoHiCan (Monument Historique Culture association nationale), Enora Juhel, Sophie Loppinet-Méo et Clémentine Albertoni

\section{OpenEdition}

Édition électronique

URL : http://journals.openedition.org/insitu/15082

DOI : 10.4000/insitu. 15082

ISSN : $1630-7305$

Éditeur

Ministère de la culture

Référence électronique

pour l'association MoHiCan (Monument Historique Culture association nationale), Enora Juhel, Sophie Loppinet-Méo et Clémentine Albertoni, «Chargé de la protection des monuments historiques : un professionnel du patrimoine au métier diversifié », In Situ [En ligne], 30 | 2016, mis en ligne le 20 juillet 2017, consulté le 30 avril 2019. URL : http://journals.openedition.org/insitu/15082 ; DOI : 10.4000/ insitu. 15082

Ce document a été généré automatiquement le 30 avril 2019.

In Situ Revues des patrimoines est mis à disposition selon les termes de la licence Creative Commons Attribution - Pas d'Utilisation Commerciale - Pas de Modification 4.0 International. 


\title{
Chargé de la protection des monuments historiques : un professionnel du patrimoine au métier diversifié
}

\author{
Designation of historic monuments, a heritage profession calling on multiple \\ skills
}

pour l'association MoHiCan (Monument Historique Culture association nationale), Enora Juhel, Sophie Loppinet-Méo et Clémentine Albertoni

1 Compter parmi son patrimoine monumental des "monuments historiques » est une spécificité française. En effet, cette expression, très présente dans notre mémoire collective, est souvent employée de manière abusive dans les médias. Elle révèle avant tout une distinction juridique. Heureusement, tout édifice, site remarquable, jardin, objet ancien n'est pas forcément un "monument historique» au sens où le cadre de sa conservation et de sa transmission aux générations futures est fixé par la loi. Il est important de replacer dans ce contexte régalien l'action des correspondants de Mérimée, devenus «chargés de la protection des monuments historiques » (CPMH). Contexte qui constitue souvent la force, mais aussi la faiblesse de l'exercice de leurs missions.

2 Les chargés de la protection des monuments historiques ${ }^{1}$ sont des agents des services déconcentrés du ministère de la Culture et de la Communication dont la mission est de «faire naître les monuments historiques $~^{2}$. Au sein des métiers du patrimoine, le CPMH se situe ainsi à la frontière entre la mission de connaissance scientifique et celle de préservation. C'est ici que vient se confronter au réel la conception intellectuelle qui a fait entrer l'édifice dans le champ du patrimoine. Reconnaître la valeur patrimoniale d'un édifice, en dehors de toute autre considération, est en effet le travail d'un chercheur, obtenir sa protection au titre des monuments historiques est en revanche un objectif qui ne peut se soustraire au contexte politique, économique et social. La procédure de protection est une «procédure ciblée et précise. Elle désigne comme un objet unique un 
édifice au statut jusqu'alors quelconque, anonyme, et est sanctionnée à son terme par la prise d'une mesure juridique individualisée et dûment motivée. Même s'ils sont des soldats de l'ombre, il existe donc bien des « acteurs » professionnels dont la mission est de concourir à la naissance des monuments historiques $»^{3}$.

Figure 1

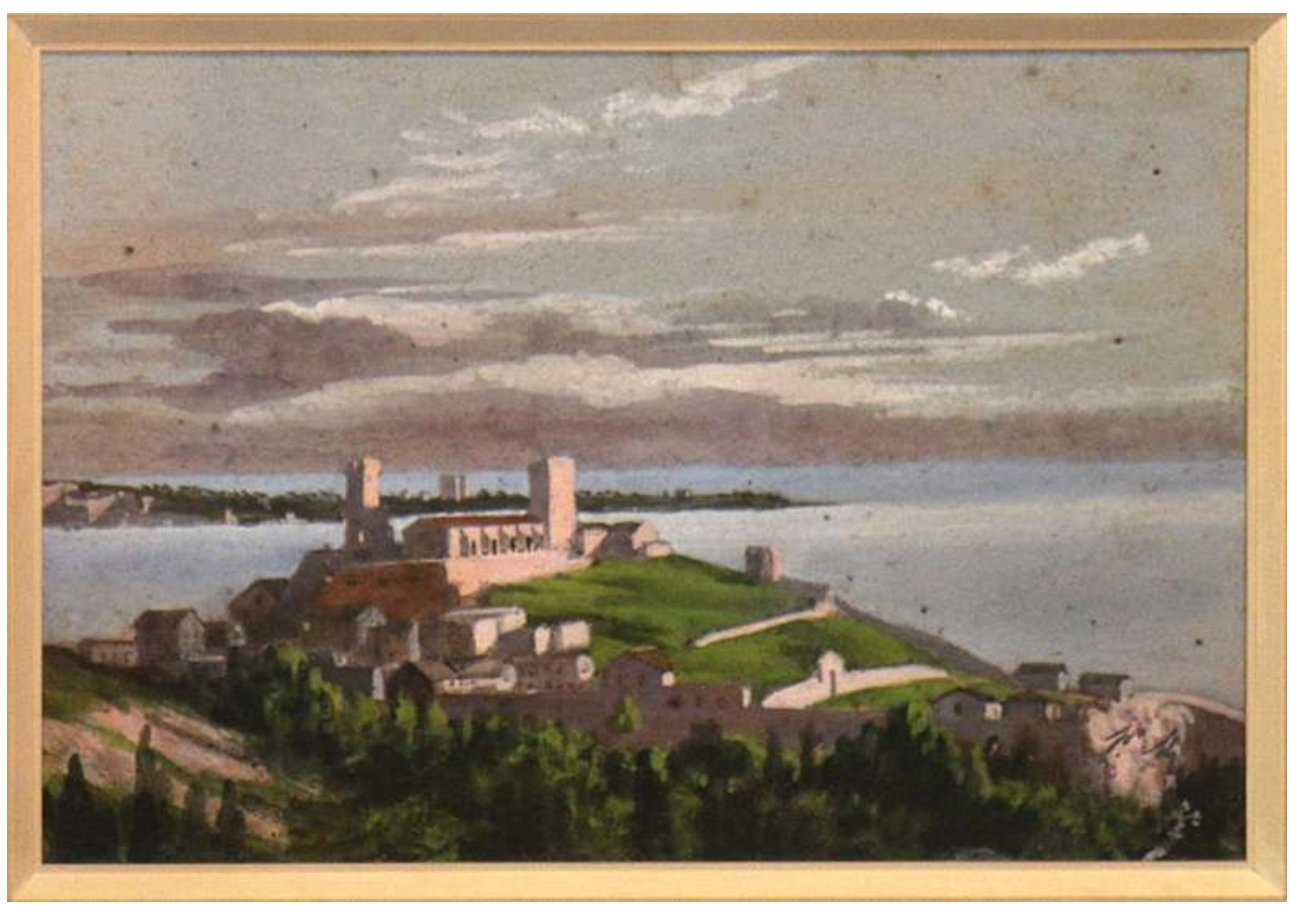

Cannes Le Suquet: gouache de Merimée.

(c) Coll. Musée d'Art et d'Histoire de Provence, Grasse - France.

3 Si la mission de ces acteurs ne s'est professionnalisée que relativement récemment, elle remonte pourtant aux prémices du service des Monuments historiques, créé par Ludovic Vitet $^{4}$, à qui succède Prosper Mérimée ${ }^{5}$ (fig. 1). Elle a été exercée de différentes manières jusqu'à aujourd'hui. Les premiers érudits chargés de repérer et documenter les édifices ont été relayés par des correspondants locaux (architectes en chef, inspecteurs, vacataires, etc.) puis, avec la mise en place, dans les années 1970, des directions régionales des Affaires culturelles (DRAC), les «documentalistes-recenseurs" des monuments historiques, aujourd'hui appelés " chargés de la protection des monuments historiques » (CPMH). Avec ce statut fixé depuis seulement une cinquantaine d'années ${ }^{6}$, c'est toute une profession qui a développé, au-delà des compétences scientifiques, techniques et juridiques, une méthodologie de travail en lien avec l'évolution de la notion de patrimoine. En effet, les hommes et les femmes qui ont exercé cette mission ont accompagné, à chaque étape, l'évolution de la politique nationale de préservation du patrimoine bâti, reflet autant de l'histoire du goût que des enjeux de territoires. Par exemple, lorsque la première liste des monuments historiques de 1840 fut publiée, les départements de l'ancienne région Poitou-Charentes ne dénombraient que 56 monuments. Tous étaient antérieurs au $\mathrm{XvII}^{\mathrm{e}}$ siècle, la moitié avait été construite à l'époque romane. Alors que le xix siècle ne semblait reconnaître comme relevant de l'intérêt public que les monuments datant de la période médiévale, ce sont aujourd'hui 
plus de 200 édifices qui sont protégés au titre des monuments historiques en PoitouCharentes, allant des périodes les plus anciennes aux dernières décennies $\mathrm{du} \mathrm{xx}^{\mathrm{e}}$ siècle ${ }^{7}$.

Cheville ouvrière de la procédure de protection au titre des monuments historiques, le CPMH est parfois le premier (voire le seul) à mener un travail d'enquête sur un édifice. Interlocuteur permanent des propriétaires, des partenaires publics et privés, des milieux universitaires et associatifs, des autres services de l'État ou des collectivités, cet acteur incontournable de la chaîne patrimoniale est également le médiateur d'un patrimoine régional qu'il connaît bien. Peu nombreux mais présents dans chaque région au sein des directions régionales des Affaires culturelles (DRAC), ils font pourtant partie des métiers méconnus de la chaîne patrimoniale. Si leur action est déterminée par leur proximité avec le territoire sur lequel ils se déplacent, la réalité de l'exercice de leur métier est aujourd'hui celle d'un réseau de chercheurs en histoire de l'architecture appliquée. La vitalité de l'association qui les réunit, $\mathrm{MoHiCan}^{8}$, illustre bien la dynamique à l'œuvre au sein de cette profession. Les CPMH s'interrogent aujourd'hui sur les outils dont ils disposent et la nature de leurs missions, à l'aune de l'évolution de la notion de patrimoine et du sens que la société lui confère. Cet article offre l'occasion d'une tentative de bilan, d'autant plus opportune à l'aube d'une nouvelle ère administrative. Face aux dernières évolutions du métier, et au moment d'une réorganisation territoriale de l'administration qui bouscule et amène à revoir les méthodes de travail, il est temps de revenir sur les fondements des missions de cet expert polyvalent du patrimoine, de s'interroger sur sa place au sein du réseau des acteurs patrimoniaux, de percevoir son rôle dans l'évolution de la notion de patrimoine et d'envisager les changements à venir.

\section{Un expert polyvalent du patrimoine}

Historien de l'architecture généraliste, amené à s'adapter en permanence, le chargé de la protection est aussi un fonctionnaire chargé de remplir une mission complexe qui mobilise, au-delà des compétences scientifiques, des savoir-faire variés et en constante évolution.

\section{De Mérimée à aujourd'hui}

\section{Héritages}

6 Si le métier de recenseur des monuments historiques ne fut créé dans l'administration qu'assez tardivement, la mission de recensement existait bien avant. Jusqu'au milieu du $\mathrm{xx}^{\mathrm{e}}$ siècle, elle consiste à réunir la documentation nécessaire à la présentation devant la Commission des monuments historiques' ${ }^{9}$. L'administration recourt alors aux inspecteurs et aux architectes des Monuments historiques ou même à des érudits bénévoles qui jouent le rôle de correspondants locaux. Le dossier de protection est fort succinct et se résume souvent à une fiche plus ou moins illustrée accompagnée de rapports (fig. 2). 


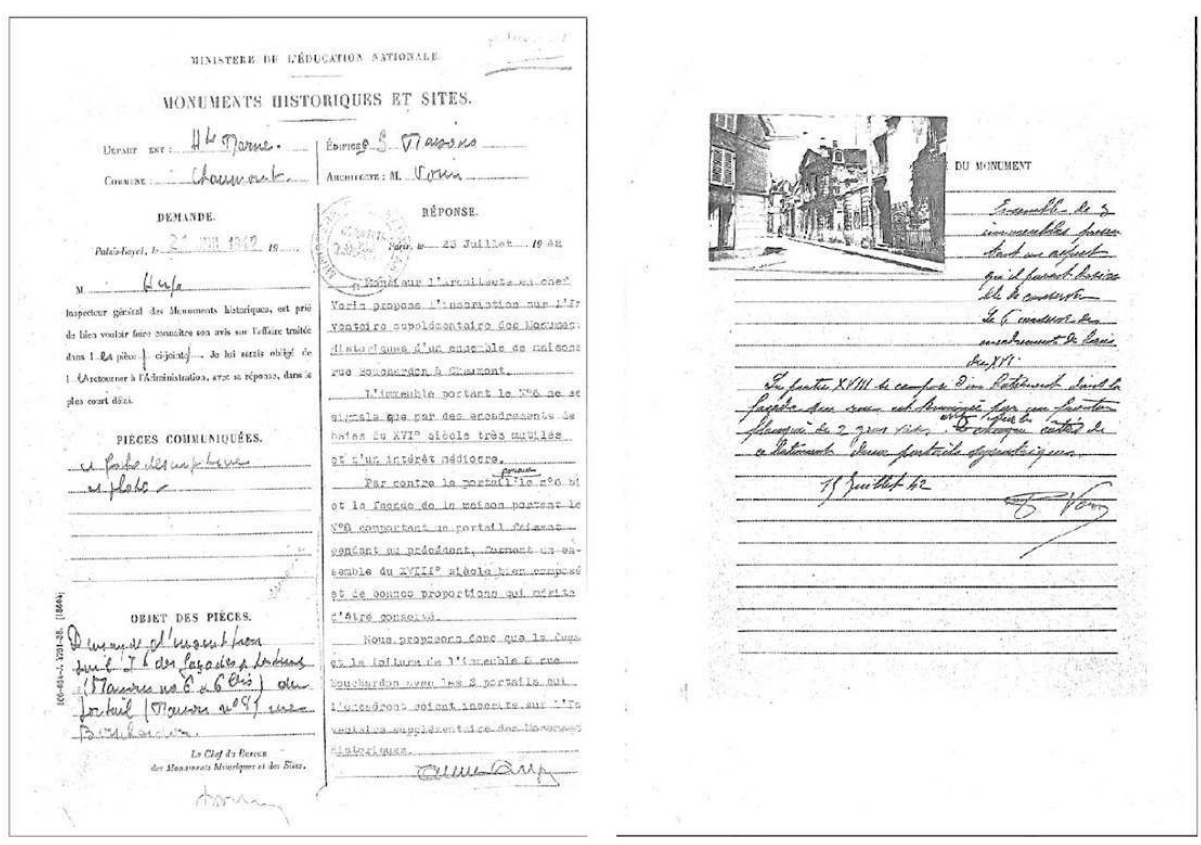

Immeubles 6 et 8 rue Bouchardon à Chaumont, inscrits le 9 août 1942. Extrait du dossier de protection comportant à gauche la demande et sa réponse et à droite l'avis de l'inspecteur général des monuments historiques.

(C) DRAC/CRMH Grand Est.

7 La mission s'individualise une première fois en 1944 avec la création du service du Recensement des monuments anciens de la France où des délégués au recensement sont chargés de constituer une documentation sur les monuments repérés afin de les inscrire au Casier archéologique de la France ${ }^{10}$. Le but du Casier est de disposer d'un outil pour mieux connaître les richesses artistiques de la France sans que ce recensement n'entraîne pour autant de servitude ${ }^{11}$. Directement rattaché à l'administration centrale, le délégué fonctionne de manière autonome par rapport aux premiers services déconcentrés, les conservations régionales des Bâtiments de France, chargées de leur côté de transmettre à l'administration centrale les demandes de protection qu'elles reçoivent au niveau local ${ }^{12}$. Dans une note adressée au ministre des Finances en 1945, le ministre de l'Éducation nationale explique que

le recensement doit au surplus amener à une meilleure utilisation des fonds mis à la disposition du service des monuments historiques. Jusqu'à présent, les classements ont souvent été prononcés au hasard des circonstances, sans méthode générale; grâce au recensement il sera loisible d'en finir avec ce manque de coordination et de déterminer rigoureusement l'ordre d'urgence des travaux de protection ${ }^{13}$.

Sur la base d'un premier travail documentaire, le délégué au recensement effectue des tournées de prospection qui donnent lieu à l'établissement de fiches. Ces dernières sont transmises chaque mois à l'architecte en chef des monuments historiques, qui donne un avis sur la mesure de protection à adopter. La fiche est ensuite envoyée au Comité de recensement des monuments anciens, à Paris.

Créé en 1947, le Comité a pour rôle de se prononcer entre une simple inscription au Casier archéologique, une inscription à l'Inventaire supplémentaire ou la transmission à la 
Commission des monuments historiques en vue d'un classement ${ }^{14}$. Le modèle de protection institué au milieu du $\mathrm{xIX}^{\mathrm{e}}$ siècle reste le même, l'administration des monuments historiques choisit le monument, demande la constitution d'un dossier et l'instruit de manière centralisée ${ }^{15}$. La mission de recensement se trouve alors à la croisée des chemins entre une entreprise d'inventaire exhaustif des richesses de la France et une opération de documentation des monuments historiques nécessaire à leur protection mais aussi aux travaux de restauration ${ }^{16}$. Ce n'est que dans les années 1960 que les missions se distinguent. Le service de l'Inventaire général des monuments et des richesses artistiques de la France est institué en 1964. Fruit de la collaboration de l'administration des Monuments historiques et des historiens de l'art, l'Inventaire cherche d'abord à se situer, entre un projet d'un inventaire qui aurait pour but d'établir la liste des édifices à protéger, soutenu par l'administration, et celui d'un inventaire à visée scientifique, indépendant de la politique de protection, souhaité par les historiens de l'art. C'est cette conception qui prévaut, et l'Inventaire général marque tout de suite sa différence par rapport au service des Monuments historiques. Son objet est beaucoup plus vaste que celui du Casier archéologique. La mission des délégués au recensement se concentre très vite exclusivement sur les futurs monuments historiques, qui doivent être documentés afin de renseigner au mieux la Commission et surtout d'informer les conservateurs et architectes du service chargés de la gestion de l'édifice.

10 Sous la pression de l'Inventaire, le recensement abandonne toute prétention à l'exhaustivité. La plupart des délégués au recensement, jusqu'alors contractuels, sont titularisés dans leur service d'origine en $1965^{17}$; très peu d'entre eux rejoignent l'Inventaire. Ils prennent le titre de « secrétaires documentalistes ». Placés sous l'autorité du conservateur régional des Bâtiments de France, ils ont pour mission de constituer la documentation des conservations régionales et de tenir leurs archives. Ce nouveau statut rattache pour la première fois la mission de protection à l'administration culturelle régionale. Cela reste cependant une mission qui relève de la documentation pure, l'établissement du rapport sur la valeur historique, architecturale et archéologique de l'édifice destiné à la Commission étant du ressort exclusif de l'architecte en chef et de l'architecte des bâtiments de France. La mission du service du Recensement évolue elle aussi. Renonçant à son ambition première, ce service centralise désormais pour les immeubles les propositions méthodiques de protection provenant des conservations régionales, conserve les dossiers de chaque monument et tient à jour la liste des monuments classés ou inscrits ${ }^{18}$. Le Comité de recensement disparaît en 1965 avec la mise en place des premières commissions régionales de l'Inventaire. Dès lors, en matière de protection, il n'est plus question d'explorer le territoire de manière systématique comme le terme de recensement, expression d'une politique ambitieuse des années 1950, pourrait le laisser croire.

11 Cette évolution lente vers la déconcentration peut se lire parallèlement à l'évolution de la politique de protection des monuments historiques dans la deuxième moitié $\mathrm{du} \mathrm{xx}^{\mathrm{e}}$ siècle. L'État, qui régissait à l'origine cette politique en fonction de sa vision de l'histoire nationale, se trouve en effet bousculé par des revendications venues de la société, qui cherche à s'approprier son patrimoine. C'est le fondement même du modèle de protection français qui est remis en cause avec, notamment, le rôle croissant que prennent les associations $^{19}$. Cette tendance culmine avec, en 1978, la création de la direction du Patrimoine et la décision du Conseil des ministres de faire de 1980 «l'année du patrimoine $»^{20}$. Du point de vue de la procédure administrative, cette période correspond 
à la déconcentration véritable de la procédure de protection avec l'instauration des COREPHAE (commissions régionales du patrimoine historique, archéologique et ethnologique), en $1984^{21}$. Pour la première fois, les propositions de protection ne sont plus envoyées directement par les services locaux à l'administration centrale qui décide ou non de leur inscription à l'ordre du jour de la Commission supérieure des monuments historiques mais elles sont présentées à une commission composée principalement d'acteurs locaux chargés de rendre un avis au préfet de région sur les mesures de protection. En 1999, les COREPHAE sont remplacées par les CRPS (commissions régionales du patrimoine et des sites), composées à parts égales de représentants de l'État, d'élus locaux et de personnes qualifiées et représentants d'associations ${ }^{22}$.

Complètement intégrés dans les DRAC, les anciens délégués au recensement sont devenus des agents qui sont en permanence en contact avec le terrain et les acteurs locaux et qui doivent faire face à un afflux de demandes. La procédure de protection est ainsi quasiment inversée, un peu plus d'un siècle après sa mise en place. À l'État centralisateur choisissant les symboles de la Nation ont succédé les demandes de protection émanant des propriétaires et de la société civile et traitées majoritairement à l'échelon local ${ }^{23}$. Le recenseur, devenu depuis "chargé de la protection", prend alors toute sa place. Cette dernière évolution sémantique, assez récente, est d'ailleurs symptomatique de l'évolution de ce métier. Alors qu'on a peut-être considéré un temps le recenseur comme un spécialiste chargé de faire les recherches documentaires et de mettre en forme un dossier, l'appellation « chargé de la protection » recouvre des missions bien plus larges et qui concernent le suivi et la gestion de l'ensemble du projet de protection, depuis la demande jusqu'à l'élaboration d'une stratégie de protection, concrétisée par la signature et la notification d'un arrêté. Revendiquée par les agents concernés comme une reconnaissance de l'évolution de leur mission, cette dénomination a fait l'objet de discussions entre l'association MoHiCan et le ministère de la Culture à partir de 2012. Elle s'est imposée définitivement dans les ordres du jour et les procès-verbaux des Commissions nationales des monuments historiques au début de l'année 2013.

\section{Qui est le chargé de protection ? Comment et pourquoi devient-on CPMH ?}

Historiens de l'art de formation pour beaucoup, ces professionnels ont acquis des compétences techniques complémentaires sur le bâti, des connaissances juridiques sur le droit du patrimoine ainsi que des connaissances administratives sur les circuits de décision relevant de la société. Leurs domaines d'intervention touchent à l'histoire, l'histoire de l'art, l'architecture, les jardins, l'urbanisme, les techniques, les sociétés ainsi qu'aux domaines de la conservation du patrimoine. Ce ne sont pas des spécialistes de tous les sujets qu'ils abordent (même s'ils sont en général experts dans une typologie ou période spécifique), ils exercent un regard transversal sur le domaine de l'histoire de l'art car ils doivent être capables de présenter des patrimoines très divers. Ce qui motive avant tout leur action est leur volonté de jouer un rôle dans la protection du patrimoine et d'ancrer leur métier au cœur d'un territoire. Ainsi, le chargé de la protection connaît avant tout son sujet par son enquête de terrain. Il pallie souvent les lacunes des archives par l'analyse in situ du bâti qui pourra l'aider à retrouver et attribuer des œuvres, ou même à enrichir le corpus des artistes. Nous pouvons citer l'exemple du château de La Borde à Vernou-en-Sologne (Loir-et-Cher), où a été découvert le seul décor subsistant de l'ornemaniste Jean $\mathrm{I}^{\text {er }}$ Bérain (1640-1711). Celui-ci, formé par le peintre Charles Le Brun, dessinateur de la Chambre et du cabinet du roi [Louis XIV] et à ce titre, chargé de créer 
des décors, a apporté une nouvelle inspiration dans les arts décoratifs à partir d'éléments "grotesques ». Son œuvre n'était connue que par ses dessins, en particulier ceux tirés de sa correspondance avec la Cour de Suède, et on pensait qu'aucun de ses décors n'avait subsisté. Le château de La Borde, édifié entre 1643 et 1645, a conservé une pièce lambrissée attribuée à Jean Bérain (1687-1688), qui se trouvait, lors de son démontage au début du xx ${ }^{\mathrm{e}}$ siècle, à l'hôtel de Mailly-Nesle ${ }^{24}$ à Paris (fig. 3). Cet apport à l'histoire des arts grâce au repérage sur le terrain se vérifie surtout pour le patrimoine dit « modeste ", qui n'a souvent donné lieu à aucune production de sources documentaires potentielles et a fortiori, n'a pas fait l'objet d'études. L'exercice du métier de recenseur diffère ainsi de l'approche du chercheur ou de l'historien d'art : il doit prendre en compte l'état sanitaire du bâtiment, son état de propriété, les menaces qui pèsent sur lui et son environnement économique et politique. Cette orientation ancre l'analyse dans la réalité de l'édifice et non seulement dans son histoire. Elle se traduit par un reportage photographique étoffé et une approche de l'évolution de sa construction qui peut s'apparenter à de l'archéologie du bâti.

Figure 3

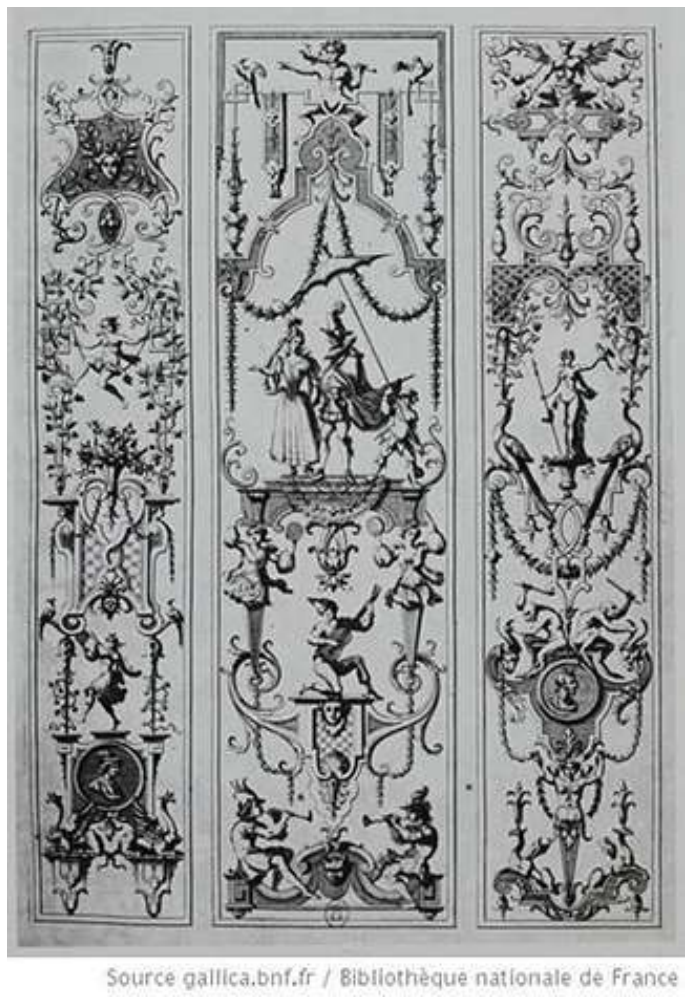

Arabesque de Jean Berain père.

(c) gallica.bnf.fr / Bibliothèque nationale de France.

Parti, au XIX ${ }^{e}$ siècle, d'une pure mission de recensement basée sur un travail de sélection de longue haleine qui faisait parfois passer l'intérêt général et national avant les réalités locales, le chargé de la protection des monuments historiques remplit aujourd'hui avant tout une mission de service public: il doit répondre aux demandes de protection provenant principalement de propriétaires, mais aussi d'associations de défense du patrimoine ou encore de spécialistes. Le contact permanent que le CPMH entretient avec les acteurs locaux privés et publics est un des aspects importants du métier souvent sous- 
estimé, de même que la concrétisation de la protection par un acte juridique qui donne du sens à la démarche scientifique. L'idéal du recensement des origines, qui visait à sélectionner de façon exhaustive les édifices exceptionnels et/ou représentatifs de notre patrimoine, demeure. Le travail par thématique, encouragé par le ministère de la Culture et de la Communication, essaie de remédier aux protections au coup par coup issues de demandes individuelles, avec le risque que celles-ci comportent d'un jugement esthétique, d'une subjectivité $d u$ "beau». Si l'opération de sélection peut paraître discutable pour des services prétendant à l'exhaustivité comme l'Inventaire, elle a le mérite d'apporter une reconnaissance concrète des édifices retenus et par là même, d'engager une dynamique de recherche dans le milieu scientifique. La démarche de protection est un engagement moral: une reconnaissance de la valeur par un acte juridique. L'arrêté est la réponse concrète de l'État à l'égard d'un patrimoine digne d'être reconnu, conservé.

Professionnel au début de la chaîne des interventions sur le patrimoine, élément déclencheur d'une prise de position, d'un engagement en faveur du patrimoine, le chargé de protection est partie prenante de la patrimonialisation sur le territoire, tâche exaltante qui demande de la réactivité et un travail de pédagogie et de suivi, notamment dans les cas d'urgence (péril). Un exemple peut être développé ici, celui des "Carrats ", à Leucate (Aude). Il illustre cette réactivité face aux menaces. Moins appréciée car moins marquée « années 1970 » que l'architecture de La Grande-Motte (Hérault), celle de Port Leucate en est pourtant exactement contemporaine. En 2010, une campagne de labellisation « Patrimoine du $\mathrm{xx}^{\mathrm{e}}$ siècle » des stations balnéaires du Languedoc créées par la Mission Racine a mis en lumière certaines constructions de la station de LeucateBarcarès, dont Georges Candilis fut l'architecte en chef à partir de 1967. À Port Leucate, deux villages de vacances et la résidence «Le village grec » ont été labellisés. En 2013, à l'annonce de la mise en vente par la Fédération des caisses d'allocations familiales du Languedoc-Roussillon du VVF Les Carrats, labellisé, la CPMH, en charge du label "Patrimoine du $\mathrm{Xx}^{\mathrm{e}}$ siècle", s'est inquiétée du devenir de cet ensemble caractéristique des vacances sociales des années 1970. En effet, le risque de démolition était important en raison du potentiel que représentent les 4 hectares de son emplacement exceptionnel sur la plage. La CRMH est intervenue pour informer le propriétaire et la commune que, en raison de l'état d'authenticité et de conservation du VVF, une protection au titre des monuments historiques devait être envisagée. La demande de classement formulée par l'association DO.CO.MO.MO France a été un appui important dans cette démarche. Ni la commune ni le propriétaire ne s'y sont opposés, dans la mesure où cela n'entravait pas la vente. Les repreneurs éventuels ont apprécié les contraintes et les avantages qu'apporterait une inscription au titre des $\mathrm{MH}$; celle-ci a été validée par un arrêté le 23 juillet 2014. Le projet de reconversion par un architecte du patrimoine est suivi par la CRMH afin que soit préservé l'aspect initial : la protection des façades et toitures pour l'ensemble permet des transformations intérieures, tandis qu'un bloc de 4 bungalows a été inscrit en totalité afin de conserver la composition par modules cubiques conçue par Candilis. La protection a clairement été un atout, à la fois pour la commune, désireuse, depuis que lui a été attribué le label "Patrimoine du $\mathrm{xx}^{\mathrm{e}}$ siècle", de valoriser l'architecture de cette station encore décriée, et pour la Fédération des CAF, très attachée à ce VVF même si elle était contrainte de s'en séparer ${ }^{25}$ (fig. 4). 
Figure 4

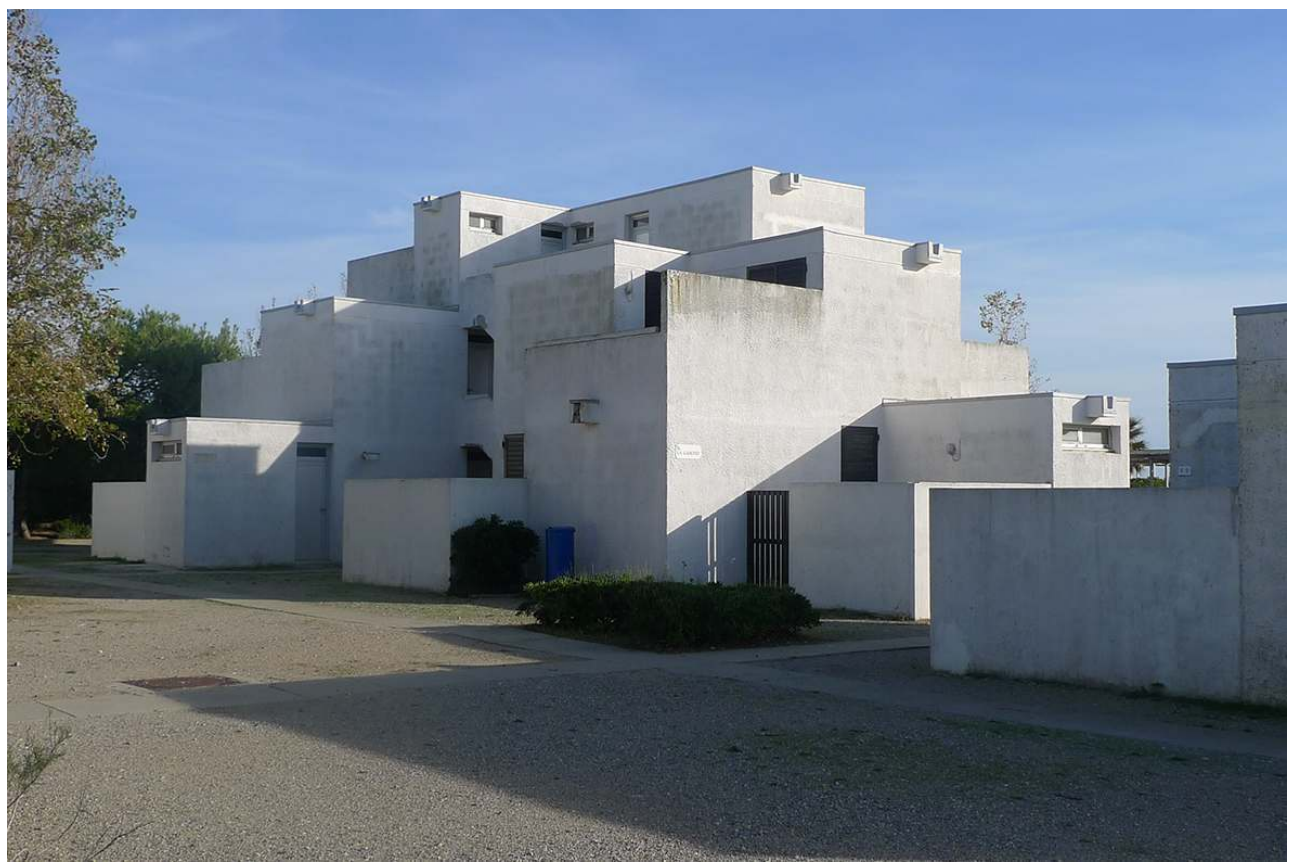

Les Carrats à Leucate : 3 collectifs.

Phot. Michèle François, 2014. (c) DRAC/CRMH LRMP.

Ce métier, situé au cœur de l'action patrimoniale, en lien avec le terrain, est jugé attractif par les professionnels. Si le délai contraint d'instruction des dossiers peut être vécu avec une certaine frustration, le travail de défricheur du CPMH lui apporte une vision globale du territoire et constitue le «fonds" sur lequel d'autres professionnels vont pouvoir s'appuyer pour en prolonger l'étude. L'implication des CPMH dans la concrétisation de la procédure de patrimonialisation, qui aboutit à la «naissance administrative » du monument historique avec l'arrêté de protection, est réelle. Mais leur nombre est insuffisant pour espérer protéger tout ce qui mériterait de l'être. L'afflux des demandes, plus ou moins intéressantes mais auxquelles la nécessité de service public oblige à répondre, ne permet pas toujours d'engager les thématiques souhaitées et un travail approfondi sur un corpus, ce qui fait naître l'impression de survoler le domaine d'étude : la distinction administrative pérenne qu'est la protection juridique au titre des monuments historiques ne concernera ainsi pas forcément l'édifice patrimonial le plus intègre, le plus authentique ou le plus signifiant.

\section{Le cœur de métier}

\section{Au cœur du métier : la constitution et le rapport du dossier de protection}

Le chargé de protection est avant tout l'auteur principal du dossier documentaire et administratif qui regroupe tous les éléments justifiant l'intérêt patrimonial de l'édifice considéré. Ce dossier est le support de la servitude. Il dévoile l'histoire de la distinction administrative du monument historique. Ce dossier connait plusieurs stades de développement, adaptés aux différentes étapes de la procédure administrative. Sa partie documentaire, la plus volumineuse, implique des recherches archivistiques, bibliographiques et de terrain, sollicitant les compétences des CPMH. 
En la matière et à titre d'exemple, la recherche d'informations sur le bâtiment de l'Espace des arts à Chalon-sur-Sâone (Saône-et-Loire) a été particulièrement compliquée en l'absence totale de publications. Elle a nécessité la consultation de fonds aux Archives nationales, à Fontainebleau. Le lancement d'une vaste opération de création de maisons de la culture sur tout le territoire français dès 1961, à l'initiative d'André Malraux, a en effet généré des échanges nourris entre les communes et l'État. Ces archives nous livrent non seulement des courriers mais aussi des plans et des photos de l'époque. Leur consultation a permis de mieux comprendre le projet de l'architecte et notamment, la fonction du parvis, devant le bâtiment, conçu à l'origine comme un espace extérieur d'exposition. Le contexte particulier du lieu d'implantation nouvellement créé avec l'assèchement du canal, dans le cadre d'une vaste opération de rénovation urbaine, a également produit de nombreuses archives locales. Enfin, les contacts téléphoniques avec le fils de l'architecte ont aidé à mieux appréhender la carrière de l'architecte Daniel Petit, architecte et urbaniste lyonnais (1907-1975), redécouvert à cette occasion, et la place du bâtiment au sein du corpus de ses œuvres. L'Espace des arts et maison des sports, y compris le parvis extérieur, partie intégrante du projet, a été inscrit en totalité par arrêté du 30 septembre 2013 (fig. 5).

Figure 5

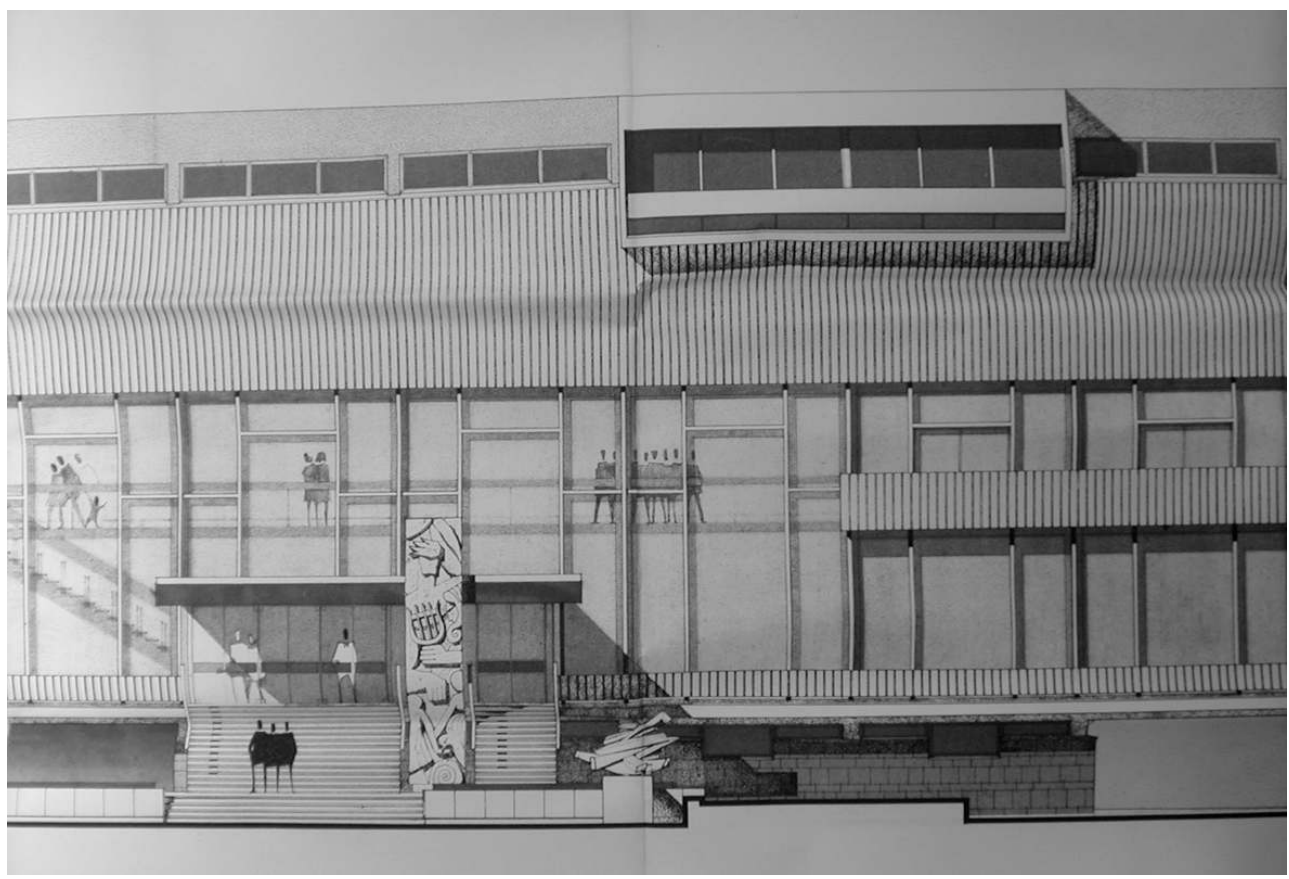

Archives nationales de Fontainebleau, répertoire 19840756, cartons 58 à 65 : façade d'entrée de l'Espace des Arts de Chalon-sur-Saône (détail).

Phot. Sophie Loppinet Méo, 2012. @ DRAC/CRMH Bourgogne - Franche-Comté.

Une fois engagée par le pétitionnaire ou par le service ${ }^{26}$, la demande de protection est présentée devant la délégation permanente de la commission régionale du patrimoine et des sites (CRPS) qui statue sur sa recevabilité, c'est-à-dire sur le potentiel patrimonial de l'édifice et la validité des motifs de la demande ${ }^{27}$. À ce stade, le CPMH rédige une courte synthèse présentée devant la commission. Même si la recherche documentaire n'est pas initiée, le CPMH doit pouvoir mettre en exergue les qualités de l'édifice aussi bien sur le 
plan historique, stylistique qu'ethnologique. Le dossier peut également être étudié par un groupe de travail réuni pour un examen thématique des demandes.

Si le dossier reçoit un avis favorable de la délégation permanente de la CRPS, le chargé de protection engage un travail d'étude scientifique sur le bâtiment proposé à la protection qui se concrétise par la réalisation d'un dossier documentair ${ }^{28}:$ recherche archivistique et bibliographique, analyse architecturale et campagne photographique sur le terrain, situation juridique et administrative de l'édifice. Ce dossier aboutit à la présentation écrite et orale d'un rapport patrimonial devant la CRPS en séance plénière, et éventuellement devant la Commission nationale des monuments historiques. La CRPS est une commission consultative placée auprès du préfet de région. Elle est composée de membres de droit et de membres nommés par le préfet de région; ils représentent l'ensemble des composantes de la société civile: fonctionnaires, élus, membres d'associations, personnalités qualifiées. Cette diversité permet d'aborder tous les aspects du dossier de protection, non seulement l'intérêt scientifique mais aussi les conséquences juridiques d'une éventuelle protection sur le territoire.

21 La description matérielle et la présentation iconographique de l'immeuble sont essentielles pour donner aux membres de la commission une idée précise de l'édifice proposé à la protection même s'ils n'ont pu se rendre sur place. Si l'exhaustivité n'est pas requise dans la visualisation des espaces concernés, il est important que les espaces significatifs soient explicités. Les documents graphiques tels qu'extraits du cadastre, plans anciens ou récents, croquis, vues anciennes et photographies actuelles accompagnent le descriptif de l'immeuble. Cette étape se conclut par une proposition de protection formulée par le $\mathrm{CPMH}$, fondée sur les arguments scientifiques et techniques relatifs à l'intérêt de l'immeuble au titre de l'histoire ou de l'art. Elle précise sur un plan cadastral l'étendue et le degré de la protection envisagée. C'est sur la proposition du rapporteur (généralement le chargé de protection) que le débat s'ouvre et que la commission fait ou non in fine une proposition de protection.

La présentation d'un dossier est assez codifiée : le CPMH présente son rapport, puis les personnes concernées par le dossier interviennent, notamment le préfet de département, le maire et le propriétaire; on lit ensuite les avis réglementaires des experts ${ }^{29}$. Le débat est conduit au sein de la commission et donne lieu à un vote (refus de protection ou avis favorable pour une ou plusieurs propositions de protection). Le " considérant », au titre de l'« intérêt d'art et d'histoire suffisant pour en rendre désirable la préservation $»^{30}$, justifie les propositions des commissions pour chaque édifice.

Le rapport du CPMH constitue donc le socle commun de connaissances des membres de la CRPS. Il permet également aux autres experts de la chaine patrimoniale de rendre leurs avis réglementaires. Ces derniers éclairent la proposition de protection en fonction de la spécialité de leurs auteurs: archéologie, Inventaire, état sanitaire, connaissance des abords, histoire de l'art et de l'architecture, etc.

24 Si l'avis de la CRPS exprime un vœu de classement, le dossier sera présenté ultérieurement par le CPMH devant la Commission nationale des monuments historiques, selon un protocole quasi identique. La présentation du dossier se fait à partir des mêmes données qu'en CRPS mais souvent la contextualisation de l'édifice est approfondie puisque l'intérêt de l'édifice doit être justifié au niveau national. La différence réside essentiellement, à cette ultime étape, dans le recueil de l'avis du propriétaire (qui doit 
être favorable) et la présentation d'éléments précis concernant la propriété foncière du monument.

Le dossier de protection s'impose alors comme une base d'informations sur l'état du bâtiment, dans l'éventualité de modifications ou de travaux. Il donne un état sanitaire précis sur lequel s'appuieront les services du patrimoine pour le contrôle scientifique et technique. Il permettra notamment d'arbitrer entre différents scénarios de réutilisation ou de restauration et de hiérarchiser la qualité des différentes parties de l'édifice. Le dossier pourra alors nourrir le travail des architectes, urbanistes, paysagistes ou aménageurs pour les études préalables aux travaux. Finalement, par ce biais, l'analyse du CPMH constitue ainsi la première pierre de la mission de contrôle scientifique et technique (CST) qui garantit la conservation des caractéristiques du monument ayant justifié sa protection. L'étude historique documentée sur l'état d'origine et les évolutions du bâti est l'outil qui sera utilisé pour arbitrer les décisions relatives à l'entretien et à la restauration du bâtiment.

\section{Le suivi administratif et juridique des protections $\mathrm{MH}$}

Dans le cadre de la réalisation d'un dossier de protection, le travail scientifique et documentaire du CPMH s'apparente donc à celui d'un chercheur en histoire de l'architecture; il s'accompagne de son nécessaire pendant administratif. Car c'est bien par la mise en œuvre du "porté à connaissance » que la servitude d'intérêt public aura une réalité opérationnelle dans les documents d'urbanisme, dans l'accompagnement des propriétaires actuels et futurs qui auront la charge du monument historique. Mais étant donné la réduction des moyens humains que connaît l'administration ces dernières années, cette mise en œuvre nécessaire de l'instruction administrative des procédures de protection pèse lourdement sur le $\mathrm{CPMH}^{31}$. La rédaction des décisions et le respect des règles de diffusion et de publicité sont en effet soumis à une attention particulière pour éviter tout contentieux.

Auteur des dossiers qu'il présente devant les commissions compétentes, le CPMH procède à l'instruction juridique des décisions préfectorales et ministérielles en matière de protection (refus et arrêtés de protection) et rédige les arrêtés de protection. C'est l'avis de la CRPS qui fonde la mesure de protection signée par le préfet de région pour les arrêtés d'inscription, et celui de la CNMH pour l'arrêté de classement émis par le ministre de la Culture. Ces arrêtés sont des actes juridiques engageant l'État et à ce titre, doivent être extrêmement précis. Ils s'accompagnent aujourd'hui systématiquement d'un plan qui ne laisse place à aucune interprétation possible quant au périmètre de protection. « Le Conseil d'État a estimé de façon constante que les mesures de protection d'immeuble au titre des monuments historiques ne sont pas des décisions individuelles. Comme elles n'ont pas non plus le caractère d'actes réglementaires, on en déduit qu'elles sont des actes particuliers, appartenant à la même catégorie que les mesures de protection de sites ou les déclarations d'utilité publique $»^{32}$.

Les CPMH n'étant pas juristes de formation, ils ne maîtrisent pas d'emblée le langage très spécifique des services de la publicité foncière et des professions juridiques sur la transmission des biens (droits indivis, réserves d'usufruit, réserves du droit de retour, actes de succession, de donation-partage, etc.). Ainsi, le déchiffrage des actes notariés, des relevés de propriété, matrices cadastrales, renseignements hypothécaires demande-t-il une certaine compréhension des codes et du jargon juridique. La rédaction des arrêtés 
donne régulièrement lieu à des difficultés supplémentaires liées aux exigences des services de la publicité foncière concernant les données relatives aux propriétaires qui doivent être spécifiées sur les arrêtés.

Le cas de la protection des tombes et cimetières et ses conséquences juridiques est particulièrement complexe: la protection porte aussi bien sur le sol (propriété communale) que sur les monuments funéraires dont les ayants droit sont souvent difficiles à identifier, la transmission d'une concession ne nécessitant pas un acte notarial. L'opposabilité aux tiers devient dès lors délicate à assurer. De même, les cimetières militaires étrangers protégés au titre des monuments historiques sont rares, leur statut d'extraterritorialité ne facilitant pas la rédaction de l'arrêté ${ }^{33}$. Enfin, citons le cas de la colonne de Montmirail qui illustre les particularités juridiques que l'on peut rencontrer : la colonne étant construite sur la limite des départements de la Marne et de l'Aisne, il a fallu un arrêté de protection signé en Champagne et un autre en Picardie.

Après la signature de l'arrêté de protection, la servitude doit être notifiée : en premier lieu, au propriétaire ; c'est la notification qui rend la décision opposable et fait courir à son égard le délai de recours ${ }^{34}$. Puis, au maire, et, le cas échéant, au président de l'établissement public de coopération intercommunale compétent en matière de plan local d'urbanisme, aux fins notamment de transcription de la servitude de protection et $\mathrm{du}$ périmètre de protection dans les documents d'urbanisme ${ }^{35}$. Une copie de cette notification est également envoyée au préfet de département et au chef de l'unité départementale d'architecture et du patrimoine. Par ailleurs, l'annexion au plan d'occupation des sols (obligatoire pour rendre la mesure opposable aux tiers ${ }^{36}$ et la publication au Journal officiel (ou au bulletin officiel du ministère de la Culture) est explicitement prévue depuis le décret de 1996. La circulaire du 26 septembre 1985 a de plus recommandé la publication des arrêtés d'inscription au recueil des actes de la préfecture de région. Le décret de 1996 prévoit enfin la publication au service de la publicité foncière de la situation de l'immeuble protégé. Celle-ci permet de garantir l'information d'éventuels acquéreurs de l'existence de la servitude de classement ou d'inscription de l'immeuble.

31 Dans certains cas, ces obligations légales représentent un effort important, surtout quand les propriétaires sont nombreux. Le classement de la nécropole protohistorique d'OrmesSimandre (Saône-et-Loire), par exemple, a été précédé par un important travail de terrain avec les 41 propriétaires des multiples parcelles (43) pour obtenir leur accord. La recherche hypothécaire, effectuée en collaboration étroite avec le service de la publicité foncière, a été compliquée par les statuts particuliers de propriété en libre pâture et en bien non délimité (BND), sans compter les successions non réglées et les indivisions complexes. Il a fallu un arrêté d'inscription préalable, le 27 avril 2007, puis deux arrêtés de classement, les 16 mai 2014 et 20 mars 2015, au gré des accords obtenus ${ }^{37}$.

Le développement des affaires de contentieux dans la procédure de protection au titre des monuments historiques a fait constamment évoluer les actes juridiques vers une plus grande précision et argumentation. Le retour sur les protections passées constitue aujourd'hui une part non négligeable des activités du CPMH: interprétation des protections anciennes, révision de protections dans le sens d'une prise en compte plus globale du patrimoine, épineuse question des radiations... Actuellement, la tendance est à la globalisation et à la mise en cohérence de la protection sur un édifice pour éviter une mosaïque de protections différentes sur un même ensemble, engendrant des difficultés de gestion des subventions et des travaux. Cette reprise de protections partielles est alors 
l'occasion de revoir la notion de patrimoine, comme ce fut le cas pour l'église SainteSégolène à Metz. Témoignage de l'architecture messine du XIII ${ }^{\mathrm{e}}$ siècle, l'église fut partiellement reconstruite à la fin du $\mathrm{xIX}^{\mathrm{e}}$ siècle, en pleine période de l'annexion allemande, par l'architecte municipal Conrad Wahn. En 1981, les parties médiévales ont été classées, alors que le reste de l'édifice était seulement inscrit. Cette protection mixte déprécie en quelque sorte la partie $\mathrm{XIX}^{\mathrm{e}}$ en lui octroyant un niveau inférieur de protection, tout en accordant une marque de reconnaissance, tardive, à l'architecture de cette époque, qui plus est allemande, en France. En 2012, alors que la Ville de Metz souhaitait restaurer l'église, on envisagea une harmonisation juridique de la protection: cette procédure, initiée pour un motif juridique, s'inscrivit alors dans une vague de redécouverte du patrimoine de l'Annexion en Moselle. Lors de sa présentation en 2013 devant la Commission nationale des monuments historiques, le dossier reçut un avis favorable à l'unanimité pour le classement en totalité "considérant, outre ses très grandes qualités architecturales, son exceptionnel intérêt pour l'histoire du patrimoine messin à l'époque médiévale et pendant la période de l'annexion allemande $»^{38}$ (fig. 6).

Figure 6

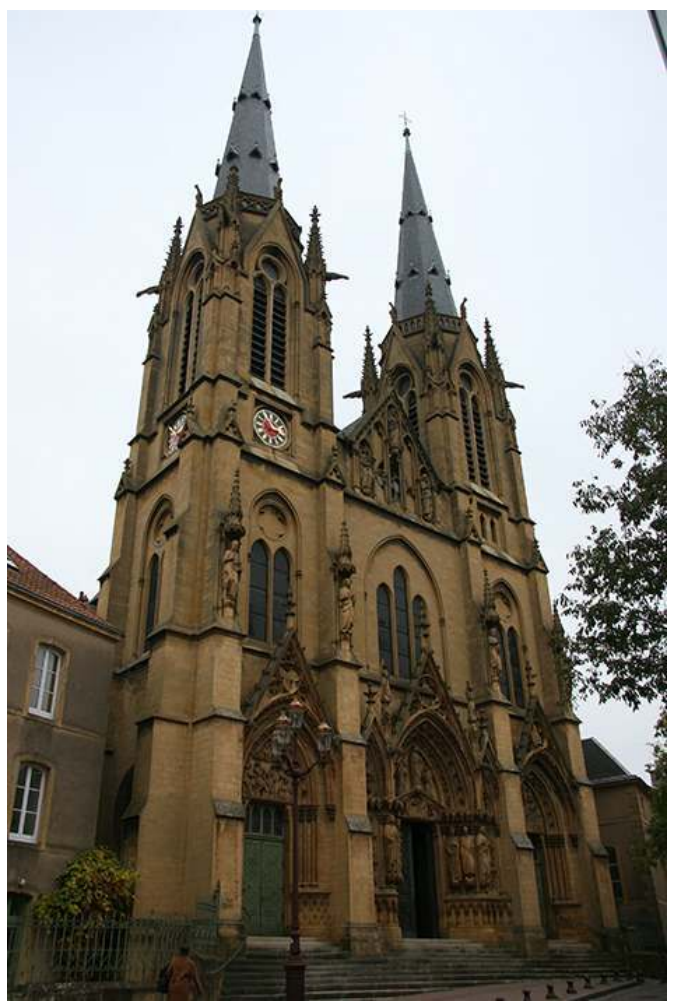

L'église Sainte-Ségolène à Metz.

Phot. Enora Juhel, 2013. @ DRAC/CRMH Grand Est.

En aval, la gestion nécessaire de la documentation, considérée comme courante, requiert son classement physique au sein du service des Monuments historiques, ainsi que l'envoi dans les structures nationales de la direction générale des Patrimoines que sont la médiathèque de l'Architecture et du Patrimoine et le service de la documentation Immeubles. Elle s'accompagne de son pendant numérique, en particulier sur les bases de données nationales. Une réflexion est en cours pour étudier les modalités d'un versement dématérialisé de ces dossiers. Enfin, chacune des étapes de la procédure, de 
l'enregistrement de la demande à la notification, donne lieu parallèlement à une saisie dans l'application nationale de gestion des monuments historiques AgrEgée, qui permet de suivre finement l'instruction du dossier. De même, le CPMH assure une veille documentaire sur les monuments historiques de son territoire. Les recours, en augmentation, pour lesquels sont demandés des mémoires en réponse, tout comme l'établissement des bilans nationaux annuels de la protection, alimentés en partie par les CPMH, qui rendent compte du travail effectué par les équipes de protection au niveau régional. Par ailleurs, le CPMH peut être amené à assurer le suivi des conventions d'exonération des droits de mutation et des agréments fiscaux, en relation avec la direction régionale des impôts.

\section{La fiabilisation des protections anciennes : révision, radiation}

Le CPMH contribue ainsi activement à l'élaboration d'une politique de protection au titre des monuments historiques. Au-delà de l'élaboration de thématiques et de leur mise en œuvre, il conçoit des méthodes et outils de révision des anciennes protections, et participe au développement de la déontologie de la protection.

La volonté de consolider ces actes juridiques a amené le ministère à envisager des campagnes de révision de protection, en particulier pour des protections anciennes imprécises parce que le descriptif de l'étendue de ce qui est protégé est trop succinct ou que les parcelles cadastrales, voire les numérotations de rue, ont changé. Le CPMH doit alors s'efforcer d'interpréter le document juridique à l'aide des archives conservées des éventuels débats des commissions de l'époque de la prise de la mesure, et parallèlement, des documents précisant les actes de propriété. À ce titre, la protection au titre des monuments historiques du monastère de la Grande Chartreuse (Isère) a fait l'objet d'une note afin d'aboutir à un "arrêté de classement interprétatif». Ce monument emblématique de l'ordre des Chartreux a été classé en 1912 avec un arrêté très sommaire: "l'ancien monastère de la Grande Chartreuse »; la note a donc porté sur l'analyse des débats qui ont précédé cette mesure, entre 1905 et 1912, le recensement des pièces produites au dossier, l'évaluation sur place de l'emprise de la protection de "l'ancien monastère de la Grande Chartreuse », la notion de « désert » et plus largement, le patrimoine cartusien, avec ses nombreuses dépendances, et a proposé la rédaction d'un arrêté interprétatif à la fois plus précis que celui de 1912 (parcelles citées) et suffisamment vaste pour que la gestion ne soit pas rendue trop complexe dans les années à venir (fig. 7). 
Figure 7

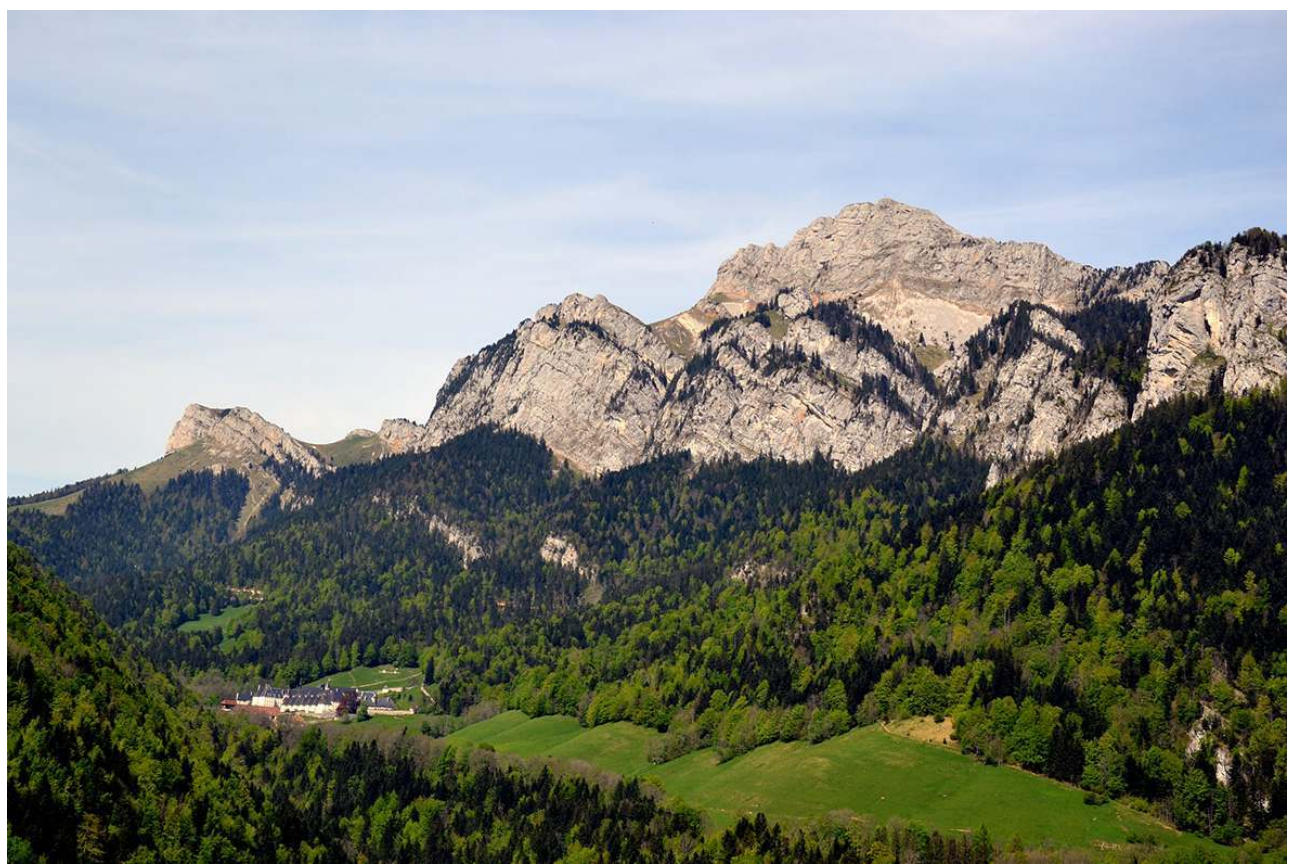

Vue du monastère de la Grande-Chartreuse et d'une partie de son désert.

Phot. Juliette Pozzo, 2015. @ DRAC/CRMH Auvergne-Rhône-Alpes.

La demande actuelle d'un contenu détaillé sur les parties protégées ainsi que sur les motifs ayant motivé la protection et d'un document cadastral accompagnant l'arrêté et précisant graphiquement l'étendue de la protection visent à éviter les contestations. Le "considérant", qui n'existait pas auparavant, prévient les éventuels argumentaires d'irrecevabilité (fig. 8). On constate d'ailleurs depuis quelques années le développement des recours amiables et contentieux sur les décisions préfectorales relatives à la protection au titre des monuments historiques, pour lesquels le CPMH est sollicité dans les mémoires en réponse. 


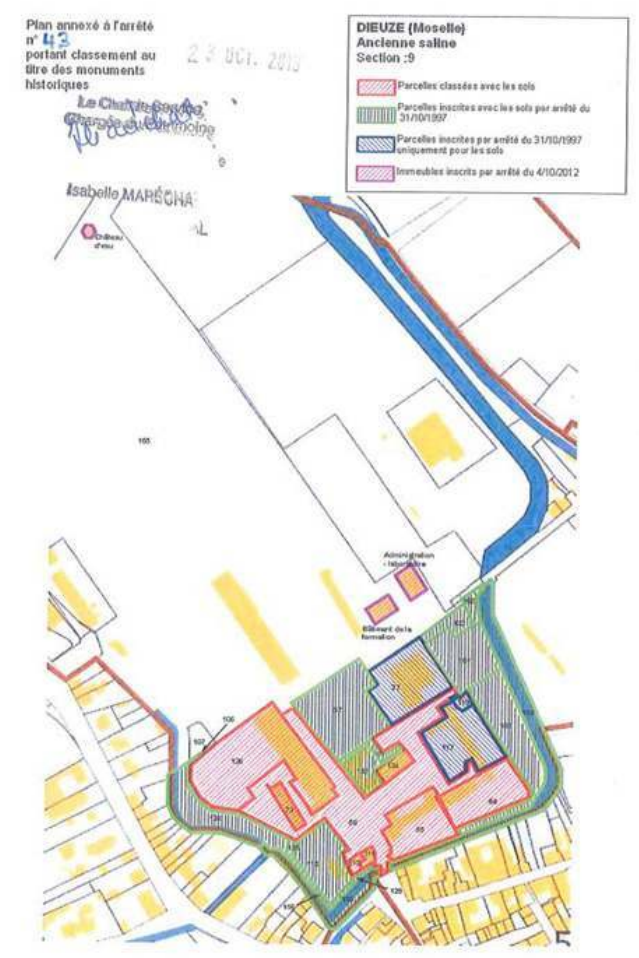

Plan annexé à l'arrêté ministériel du 23 octobre 2013 portant classement au titre des monuments historiques de l'ancienne saline de Dieuze.

(c) DRAC/CRMH Grand Est.

D'autres cas de reprises d'arrêtés ont trait au déclassement ou à la radiation de l'inscription d'un immeuble pour des édifices entièrement ou en majeure partie détruits soit par faits de guerre, soit à la suite de démolitions légalement autorisées. La démarche répond alors au parallélisme des formes et est la même que pour un arrêté de protection au titre des monuments historiques.

L'évolution de la doctrine a aussi reconsidéré la question de la distinction entre meubles et immeubles. Ainsi, certains arrêtés de protection au titre des objets mobiliers concernent des biens aujourd'hui considérés comme immeubles par nature, comme les vitraux ou les peintures murales. Un nouvel arrêté doit alors être pris avant abrogation de l'arrêté objet mobilier. Le cas de la maison métallique Duclos à Oissel (Seine-Maritime) a suscité des interrogations sur son statut, immeuble ou meuble. Édifiée selon le brevet de construction déposé en 1890 par Bibiano Duclos, cette maison est démontable et prévue pour être produite en série. Elle a plusieurs fois changé d'emplacement jusqu'en $1935^{39}$. La CRPS a longuement débattu du type de protection à envisager avant de finalement trancher en 2012 par une inscription au titre des immeubles. Ce dossier a été présenté à la CRPS avec ceux de deux autres maisons métalliques des années 1930 de dimensions plus modestes, démontables elles aussi : un pavillon métallique des Forges de Strasbourg pour la SNCF et une maison "Tout acier» de Fillod pour des chantiers navals. La maison Duclos, plus ancienne, est un édifice beaucoup plus vaste, à étages, qui s'apparente plutôt à la typologie architecturale des maisons de maître. Lors des débats, l'ACMH a fait observer le caractère modulable et démontable de ces maisons métalliques. Après avoir 
souligné l'intérêt technique de cette architecture, le CRMH précise que la réglementation ne s'oppose pas au déplacement d'un MH. Pour le DRAC, il s'agit d'un mode de construction qui n'est ni éphémère ni provisoire. Ces maisons sont une réponse aux problèmes de logement et d'habitat, elles sont arrivées dans un tissu urbain particulier, lié à une histoire industrielle, ce qui n'est pas anodin. Finalement, trois avis favorables assortis de projets de PPA ont été émis par la CRPS. Inscrites, les maisons n'ont pas été déplacées, seule la maison des Forges de Strasbourg est en cours de restauration (fig. 9).

Figure 9
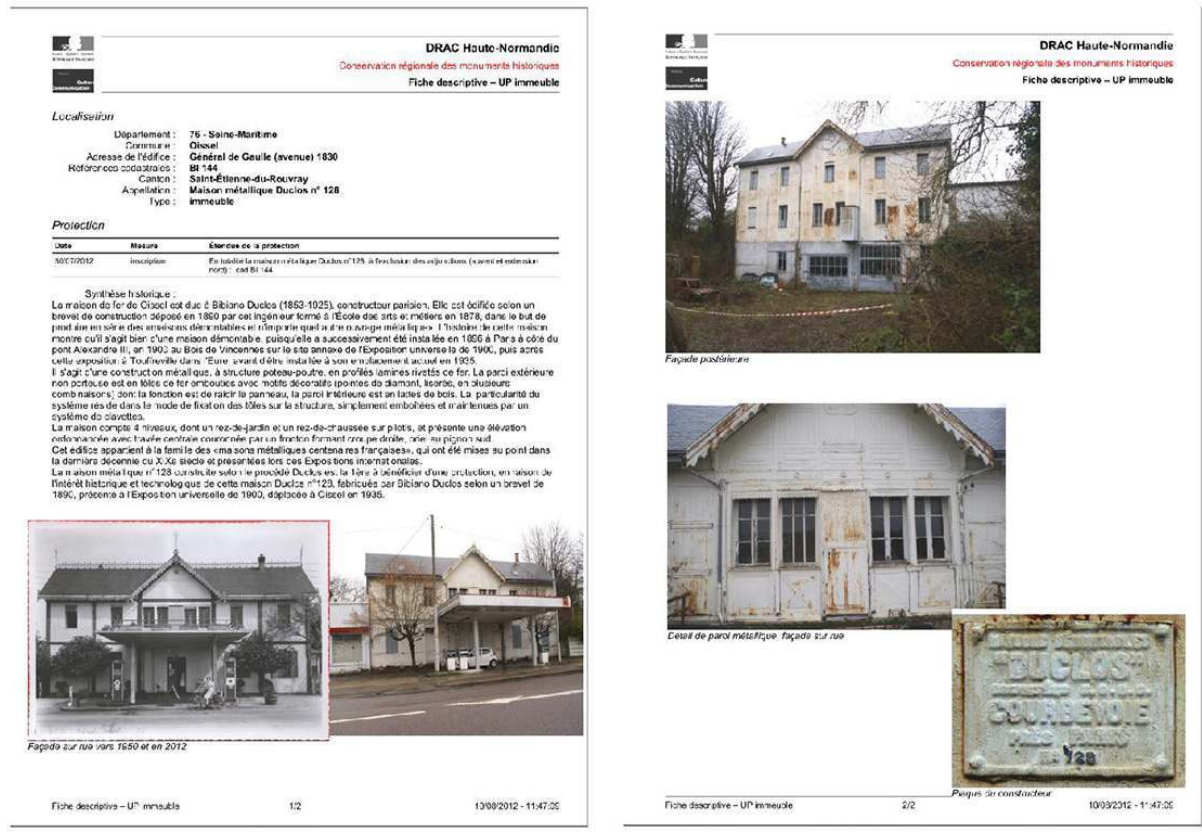

Maison Duclos : fiche descriptive UP immeuble.

Phot. Élisabeth Wallez, 2012. (C DRAC/CRMH Normandie.

Si le CPMH est un historien de l'architecture dans l'âme, en a-t-il encore les moyens, face à la multiplicité des missions dont il a la charge ? Sa spécificité liée à la protection est battue en brèche par les tâches nouvelles qui lui sont confiées mais qui enrichissent sa connaissance du domaine patrimonial. Placé comme il l'est en première ligne, de fait, le CPMH a su organiser son travail en interface avec les autres métiers du patrimoine et développer un réseau capable d'initier, d'accompagner et de relayer sa démarche. $\mathrm{La}$ maitrise de la méthodologie documentaire dans le domaine de l'histoire de l'architecture lui permet notamment de nourrir ses propres travaux mais aussi ceux des architectes, urbanistes, paysagistes ou aménageurs en quête d'information ${ }^{40}$. 


\section{La place du CPMH dans les réseaux patrimoniaux}

\section{Diversité des acteurs, diversité des points de vue}

\section{Des partenaires institutionnels privilégiés}

40 L'acteur par excellence avec qui le chargé de protection collabore le mieux est sans doute le service de l'Inventaire général du patrimoine culturel. Producteur d'un avis en commission régionale, celui-ci apporte au dossier les données de corpus indispensables pour faire ressortir l'intérêt d'un édifice. Bien conduits, les rapports entre ces deux services, chargés l'un d'une mission de protection, l'autre d'une mission d'inventaire, peuvent donner des résultats fructueux, notamment en ce qui concerne le repérage, même quand ils sont confrontés aux réalités du terrain et à l'absence de volonté des propriétaires. Ainsi, en Lorraine, une collaboration étroite entre le service de l'Inventaire et celui des Monuments historiques autour du patrimoine juif a abouti à la protection de plusieurs synagogues et cimetières. Lors de ce travail, la commission régionale s'est appuyée sur les connaissances du service de l'Inventaire pour sélectionner les édifices les plus représentatifs ou exceptionnels. Certains n'ont en revanche pas pu être protégés en raison du refus des propriétaires. La complémentarité entre chercheurs de l'Inventaire et CPMH est alors ici particulièrement visible : tous les éléments du patrimoine ne sont pas appelés à bénéficier d'une protection au titre des monuments historiques mais le travail d'étude et de valorisation mené par l'Inventaire contribue aussi à leur sauvegarde ${ }^{41}$.

41 Lors de la constitution du dossier de protection, le CPMH rencontre différents cas de figures: pour un sujet bien connu, déjà traité dans diverses publications, il aura facilement recours aux ressources documentaires à sa disposition; pour un sujet peu étudié ou sur lequel la recherche est en cours, il a tout intérêt à solliciter les personnes ressources qu'il rencontrera parmi les érudits locaux (sociétés savantes, associations...) et a fortiori, les universitaires. Ainsi des experts sont-ils régulièrement invités lors des CRPS afin d'éclairer les débats. Ces coopérations peuvent susciter une émulation scientifique autour d'un sujet et, par là, entraîner une avancée pour la recherche scientifique autant que pour le patrimoine.

L'exemple que nous retiendrons ici est celui d'Audressein, village des Pyrénées ariégeoises qui possède, avec l'église Notre-Dame de Tramezaygues, un édifice classé monument historique et inscrit sur la liste du patrimoine mondial de l'Unesco au titre des " chemins de Saint-Jacques ». Mais en 2002, c'est un autre édifice de la commune, situé à proximité, qui a retenu l'attention de la DRAC, à la suite de la demande de protection d'une batteuse fonctionnant à l'énergie hydraulique grâce à un système installé sur un bief en aval d'un moulin et d'une scierie. L'inventeur de ce dispositif, aïeul des propriétaires demandeurs de la protection, était un «notable » du village. Au moment de la constitution du dossier, la première question qui se posait était celle de l'existence éventuelle de dispositifs similaires en France, voire dans d'autres pays européens. Des échanges avec le conseiller pour l'ethnologie de la DRAC, suivis de contacts avec divers organismes spécialisés dans l'étude et la connaissance du patrimoine rural (musée des Ruralies de Niort, conservatoire du machinisme agricole de Chartres, ingénieurs agronomes, responsables de «musées paysans»), aboutirent à une conclusion assez étonnante: ces spécialistes découvraient l'existence de ce type d'aménagement et la batteuse hydraulique d'Audressein constituait apparemment un unicum. La batteuse a été 
inscrite au titre des monuments historiques par arrêté du 17 mars 2003 et au titre des objets mobiliers pour sa partie mécanique, le 27 décembre 2002. Ayant bénéficié de l'aide de la Fondation du patrimoine pour sa restauration, elle est ouverte régulièrement au public pour de nombreuses visites guidées et animations ${ }^{42}$ (fig. 10).

Figure 10

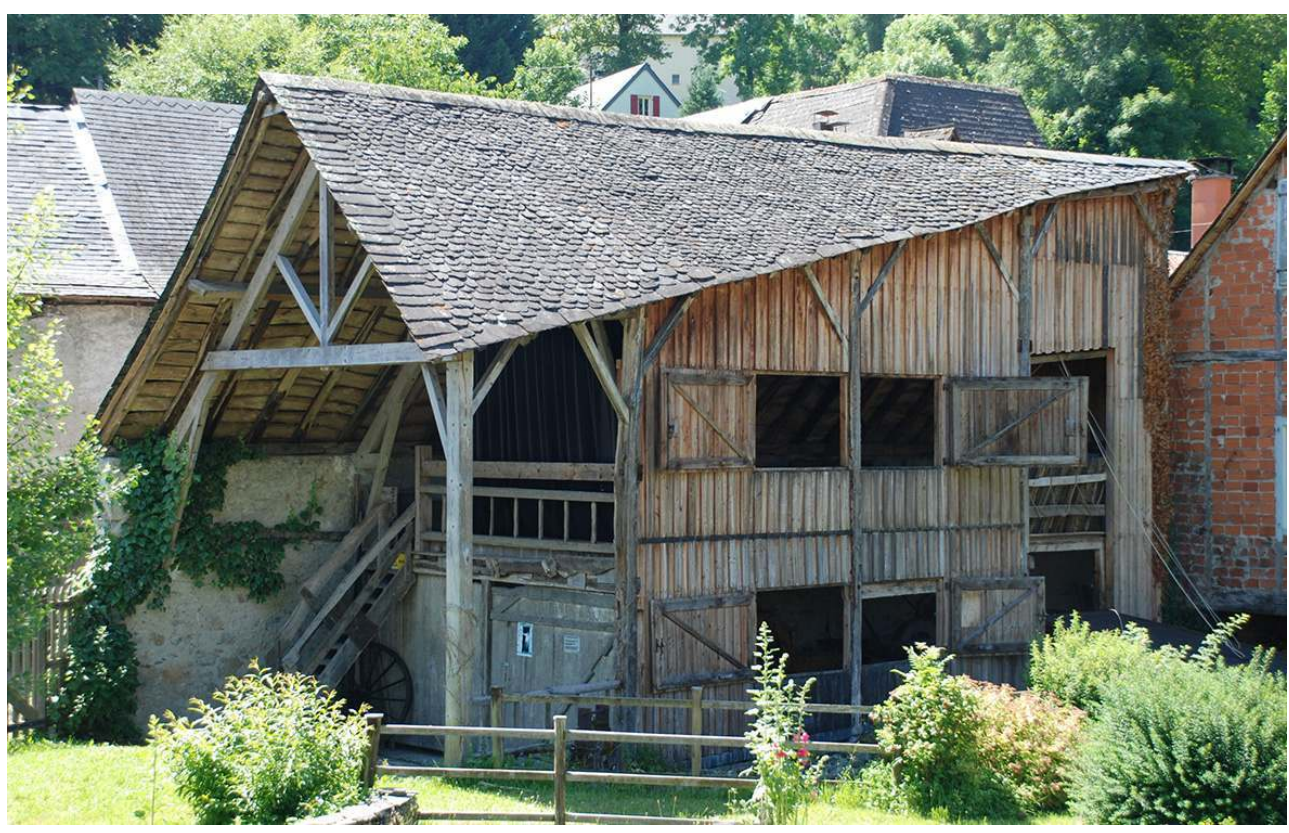

Batteuse hydraulique à Audressein : vue rapprochée de la façade au-dessus du canal avec ses ouvertures destinées à l'aération et son pont-levis.

Phot. Georges Gonsalvès, 2016. (c) DRAC/CRMH LRMP.

Le chargé de la protection se trouve à l'interface des acteurs du champ scientifique et professionnel $\mathrm{du}$ patrimoine, chargés de faire vivre le monument à partir de la protection, qu'ils soient architectes, conservateurs des monuments historiques, CAOA (conservateurs des antiquités et objets d'art), ABF (architectes des bâtiments de France), ingénieurs ou techniciens du patrimoine. Une protection réussie implique souvent ces acteurs dès le début de la procédure afin de mener à bien le projet de protection. C'est grâce à une collaboration renforcée entre la $\mathrm{CPMH}, \mathrm{l}$ 'ABF, le propriétaire et les deux communes impliquées, concrétisée par la présentation commune du dossier, que la CRPS de Lorraine a attribué en 2013 le label « Patrimoine du xx $x^{\mathrm{e}}$ siècle » aux usines et aux cités ouvrières de Bataville (Moselle), et a rendu un avis favorable à l'inscription au titre des monuments historiques des principaux bâtiments et au périmètre de protection adapté (PPA) englobant l'ensemble du site établi par l'ABF. La protection et la gestion urbaine du site de Bataville montrent comment les outils et les acteurs peuvent s'accorder pour la protection d'un site complexe ${ }^{43}$ (fig. 11, 12, 13). 
Figure 11

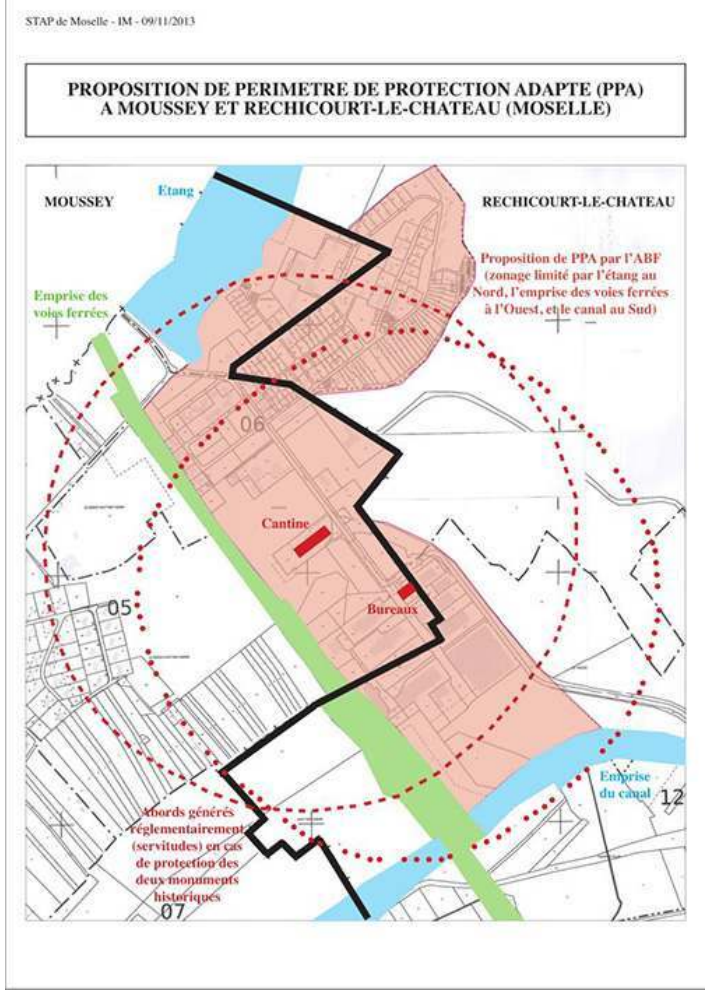

Plan du PPA de Bataville, 2014

(c) DRAC/CRMH Grand Est.

Figure 12

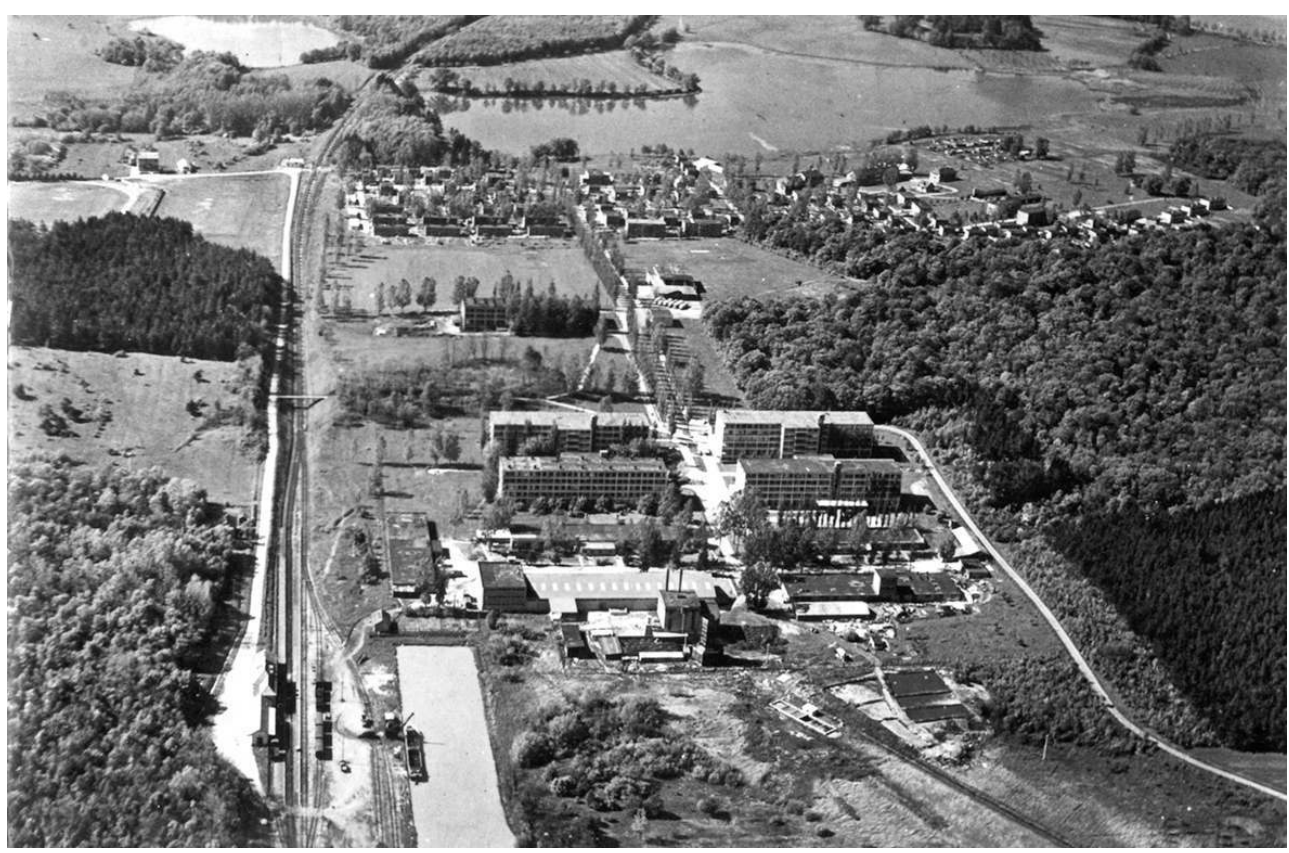

Vue aérienne de Bataville vers 1950.

(c) Archives départementales de Moselle. 
Figure 13

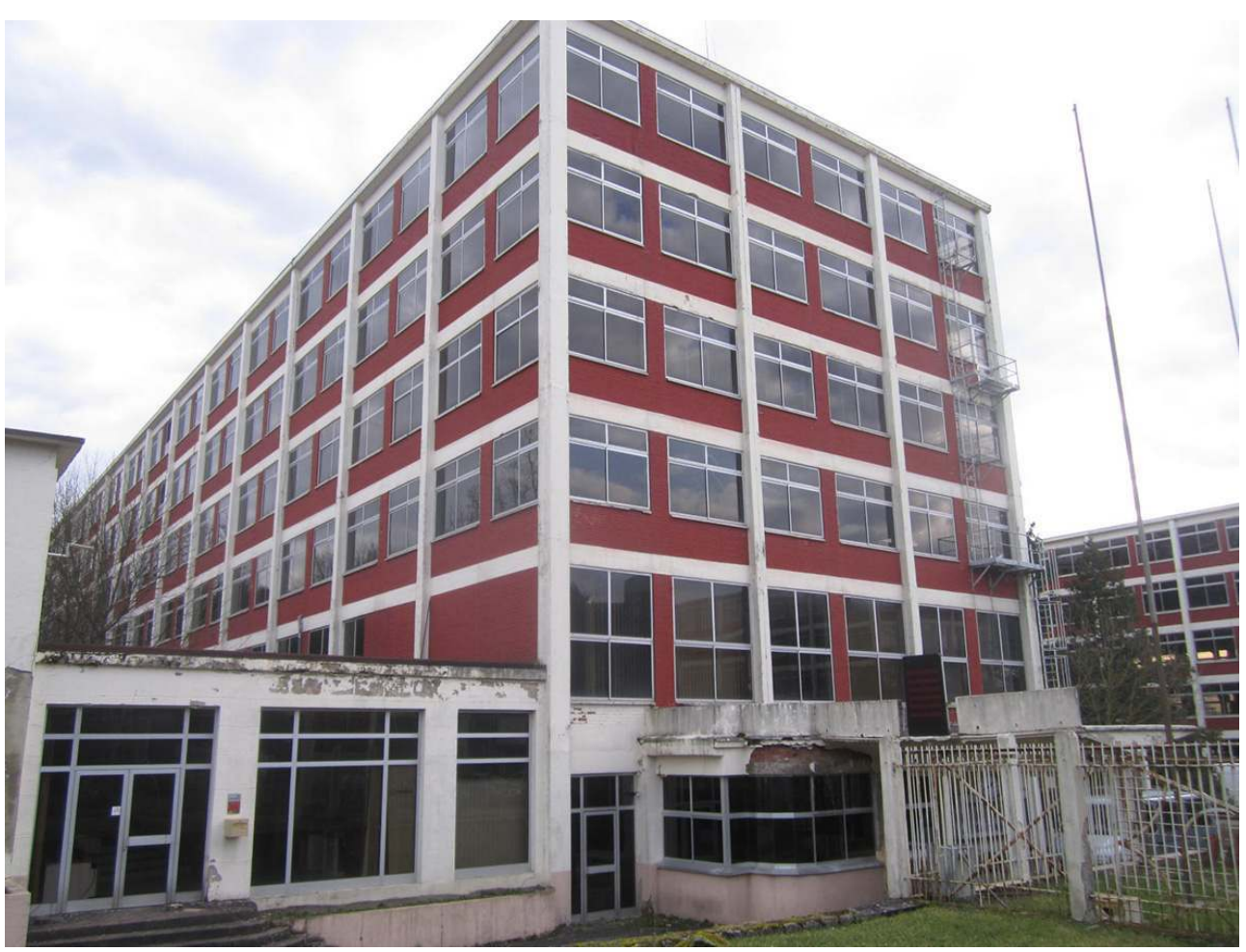

Bâtiment de l'administration à Bataville.

Phot. Enora Juhel, 2013. (c) DRAC/CRMH Grand Est.

\section{Le rôle des élus et des associations}

44 À côté de ces acteurs professionnels, le chargé de la protection est aussi confronté à la société civile qui cherche à se réapproprier, à juste titre, son patrimoine. C'est cette collaboration étroite avec les acteurs locaux qui distingue peut-être le plus l'activité du CPMH des autres métiers de documentation du patrimoine. Le monument n'est pas hors sol et le dossier de protection ne peut faire abstraction du contexte d'implantation de l'édifice. Localement, les associations de protection et de valorisation du patrimoine et les sociétés savantes apparaissent alors souvent comme les interlocuteurs de premier plan. Elles assurent à la fois une fonction de médiation et de veille sur le terrain. En Alsace, par exemple, la Société pour la conservation des monuments historiques d'Alsace (SCMHA) ${ }^{44}$ est une association ancienne et dynamique qui joue un rôle essentiel identifiant les édifices remarquables, soutenant la politique de protection des services de l'État et servant de relais auprès des propriétaires privés qui ont un monument historique ou qui souhaitent faire protéger un bien. Créée pour assurer la préservation et l'étude du patrimoine archéologique et architectural de l'Alsace, elle s'est acquittée de cette mission dès 1855 grâce à un vaste réseau de correspondants sous le Second Empire puis au temps du Reichsland, entre 1870 et 1918. Son action a été relayée et amplifiée par la création progressive par l'État des services patrimoniaux à la fin du XIX et au xxe siècle, services avec lesquels la Société a toujours entretenu une étroite collaboration. Elle a aussi donné naissance au début $\mathrm{du} \mathrm{xx}^{\mathrm{e}}$ siècle à de nombreuses sociétés locales, en particulier basrhinoises, qui lui étaient affiliées à l'origine et qui ont repris ses objectifs à l'échelle de leur canton ou arrondissement. Son président actuel, Guy Bronner, membre depuis 1986 
des commissions patrimoniales ${ }^{45}$, porte à ce titre un discours expert fédérateur qui s'appuie sur l'expérience et les compétences de l'association.

Les associations patrimoniales sont également souvent le relais et le soutien des propriétaires. Les trois principales associations nationales, Maisons paysannes de France, Vieilles maisons françaises et la Demeure historique, éditent chacune une revue qui ouvre le débat sur des sujets de société patrimoniaux et apporte des témoignages sur des acteurs emblématiques du patrimoine. La Fondation du patrimoine est chargée plus particulièrement d'aider financièrement les propriétaires pour les travaux sur des édifices protégés ou non. Elle coopère avec les services de la DRAC, qui font d'ailleurs partie du comité technique. Dans ce contexte, le CPMH peut être amené à ouvrir une procédure de protection en lien avec un projet de travaux.

De même, les communes peuvent être amenées à jouer un rôle moteur dans la protection, constituant un vrai levier pour une démarche efficace et réussie, en assurant le relais pédagogique et politique de la démarche du CPMH. Cette volonté politique, relayée par des actions en faveur de la valorisation du patrimoine (publications, visites guidées, événements à portée patrimoniale), constitue un premier pas susceptible d'engendrer une dynamique locale pour la préservation du patrimoine ${ }^{46}$.

\section{La place des propriétaires}

Les propriétaires, en particulier les propriétaires privés, et la nature des relations que l'on entretient avec eux, sont très divers, à l'instar des types d'édifices sur lesquels les CPMH ont à se pencher. Ici, le chargé de la protection peut jouer un rôle crucial: interlocuteur privilégié, il est celui qui entre dans l'intimité du propriétaire qui ouvre alors sa demeure à un parfait étranger. La procédure de protection, qui peut prendre des années, nécessite bien souvent des visites répétées qui permettent d'affiner la proposition de protection. L'accord du propriétaire, indispensable pour le classement, plus que souhaitable même s'il est facultatif pour l'inscription, est bel et bien la « clef de voûte » de la protection et découle bien souvent de la confiance établie entre le propriétaire et le $\mathrm{CPMH}$. Les réticences d'un propriétaire sont fréquemment à l'origine d'une absence de protection au titre des monuments historiques sur des monuments reconnus depuis longtemps dans l'histoire de l'architecture. Cette spécificité montre bien l'importance que revêtent les contacts personnels dans le travail du CPMH. Parmi les acteurs de la chaîne patrimoniale, chercheurs ou associations, il peut rencontrer plus de difficultés sur certains sujets car il est associé à un arsenal réglementaire difficile à défendre, surtout dans un contexte de baisse des dotations de l'État. Lorsque les intéressés soutiennent le projet, la visite du CPMH est l'occasion d'une mise à plat, d'un nouveau regard des propriétaires et des élus sur leur patrimoine. Le CPMH initie et favorise cette nouvelle vision ; il accompagne les propriétaires dans leur démarche de gestion et de connaissance de leur propriété, protégée ou non au titre des monuments historiques. De bonnes relations au moment de la protection augurent généralement de bonnes relations avec les services de l'État dans la suite de la vie du monument historique.

Le café du Rocher à Marsannay-la-Côte (Côte-d'Or) offre ici un exemple pertinent. En effet, la redécouverte de ce bâtiment, un peu oublié, a été à l'origine d'une démarche patrimoniale militante auprès des commissions dans le cadre de l'instruction, mais aussi pédagogique auprès des propriétaires et des gérants. Le travail accompli sur le terrain pour faire prendre conscience de l'intérêt de ce décor intérieur de peintures et 
d'enrochements, qui constitue un unicum, associé à une activité de café depuis presque deux cents ans, a démontré l'opportunité de le conserver, d'autant qu'il peut également être source d'attractivité touristique et commerciale. Le café est inscrit depuis le 15 septembre 2015 et l'arrêté de classement est en cours (fig. 14).

Figure 14

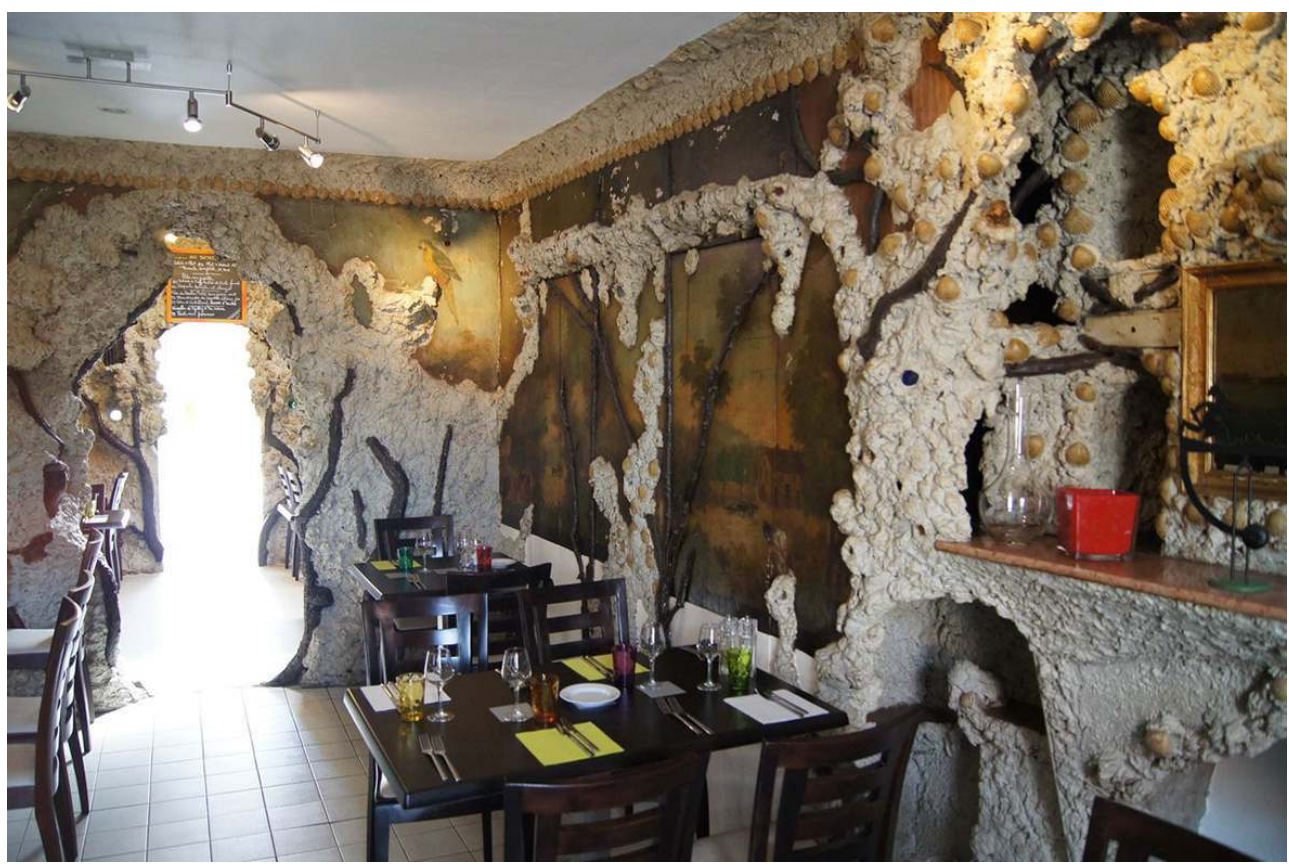

Café du Rocher à Marsannay-la-Côte.

Phot. Sophie Loppinet Méo, 2016. @ DRAC/CRMH Bourgogne - Franche-Comté

\section{Les moyens d'action}

49 On le voit, la protection au titre des monuments historiques est aujourd'hui l'aboutissement d'une dynamique dont les acteurs sont multiples. Elle ne peut plus se contenter d'une "note synthétique " rédigée par un correspondant local et visant à convaincre le service des Monuments historiques «de Paris " de l'impérieuse nécessité d'une protection. La procédure de protection, si elle reste une procédure administrative, ne se limite pas non plus au suivi de règles énoncées dans des circulaires. Afin d'assumer sa mission de préservation et de transmission des édifices témoins de notre histoire, le CPMH doit trouver de nouveaux moyens d'action.

\section{Mettre en œuvre une réelle politique raisonnée de gestion des demandes de protection}

Face au flux de demandes de protection, il est de plus en plus difficile pour l'État de mener une politique de protection visant à sélectionner des thématiques. Le CPMH s'est toujours efforcé, malgré tout, de trouver des moyens d'action qui visent à donner plus de cohérence aux protections. La tâche de la délégation permanente est ici cruciale. Plus qu'un préalable à la présentation en commission plénière, le passage devant cette instance est certes l'occasion d'effectuer un tri parmi les demandes, mais surtout d'établir une hiérarchie entre elles et de susciter la naissance de thématiques de protection. Son 
avis, notifié officiellement, contribue aussi à attirer l'attention des propriétaires ou de la société civile, dans le cas de protections souhaitées par l'administration sans demande particulière.

51 Ainsi, la gestion des demandes de protection courantes ne doit-elle pas empêcher le CPMH de mener des campagnes thématiques. Cependant, s'il est intéressant de faire un travail sur un corpus, il est toujours plus délicat de convaincre les propriétaires réfractaires. Afin d'éviter de perdre temps et énergie sur des sujets qui ont peu de chances d'aboutir en commission, il est souvent plus utile de s'appuyer sur des dynamiques locales, même s'il faut des années avant d'obtenir un accord. Ces travaux sont alors bien souvent menés en concertation avec différents acteurs au sein de groupes de travail animés par le CPMH et sont généralement très fructueux.

52 L'une des dernières campagnes thématiques lancée par la direction générale des Patrimoines du ministère de la Culture et de la Communication porte sur les phares. En effet, l'automatisation des équipements et les technologies de positionnement par satellite ont conduit le ministère de l'Écologie et celui de la Culture et de la Communication à se pencher sur le devenir de ces édifices. C'est ainsi qu'a été initiée une campagne de protection au titre des monuments historiques sur le territoire national, y compris l'outre-mer, entre 2010 et 2012, donnant lieu à deux commissions nationales consacrées à cette thématique. Dans le cadre d'une campagne de protection d'un corpus disséminé sur l'ensemble des côtes françaises, les chargés de protection allaient relever le défi d'une démarche délicate et complexe. Les phares sont à l'évidence les ponctuations d'une seule et même continuité littorale où, comme il est de règle, de nouveaux dispositifs ont sans cesse supplanté les anciens dans le souci d'une efficience accrue ou pour contrer les assauts de la mer, et ce, depuis 1825 . Une approche systématique imposait de réunir de solides éléments de compréhension - entreprise facilitée par l'abondance et la qualité des archives du service des Phares et Balises - pour analyser l'architecture des phares à proprement parler, les optiques avec leurs mécanismes adjacents, les aménagements de vie (logement, local technique) ainsi que les éléments périphériques (jardin, mur de clôture, plate-forme, quai ou terrasse). L'enjeu de la protection résidait aussi dans la manière d'appréhender à la fois l'histoire des balises maritimes et terrestres, l'évolution des constructions et des technologies et la grande diversité des réponses apportées par les ingénieurs des Phares et Balises, selon l'époque et le lieu, pour constituer " une chaîne lumineuse " sur le littoral. Méthodique, la démarche de protection se fonda sur une classification architecturale en trois catégories: les phares à tour centrée sur soubassement carré selon le modèle de Léonce Reynaud, les maisons-phares et les phares à tour isolée, sur terre ou en mer, en fer ou maçonné. À chacun de ces trois ensembles se superpose un segment temporel: décennie 1830 , seconde moitié $d u x{ }^{e}$ siècle et $x^{e}$ siècle. Cette campagne thématique interministérielle, associant administration centrale et services déconcentrés et réunissant des compétences pluridisciplinaires, démontra pourquoi, dans un contexte devenu très contraint, une des réalités tangibles du patrimoine national jusque-là sous-représentée devait bénéficier du statut de monument historique. Ce travail ambitieux a été l'occasion pour les Éditions du patrimoine de publier l'ouvrage Phares: monuments historiques des côtes de France ${ }^{47}$, paru en 2013. Ainsi, dans la continuité de ce travail, sur proposition de la CRPS de Bretagne, le préfet de région a, le 31 décembre 2015, inscrit au titre des monuments historiques 14 nouveaux phares en Bretagne. Ces protections font suite au classement, entre 2010 et 2013, de 13 phares et anciens phares de Bretagne ${ }^{48}$ et s'inscrivent dans une démarche concertée 
d'étude, de préservation et de valorisation des phares mise en œuvre par les services du ministère de l'Écologie, du Développement durable et de l'Énergie (subdivision des Phares et Balises) et de la DRAC Bretagne ${ }^{49}$ (fig. 15).

Figure 15

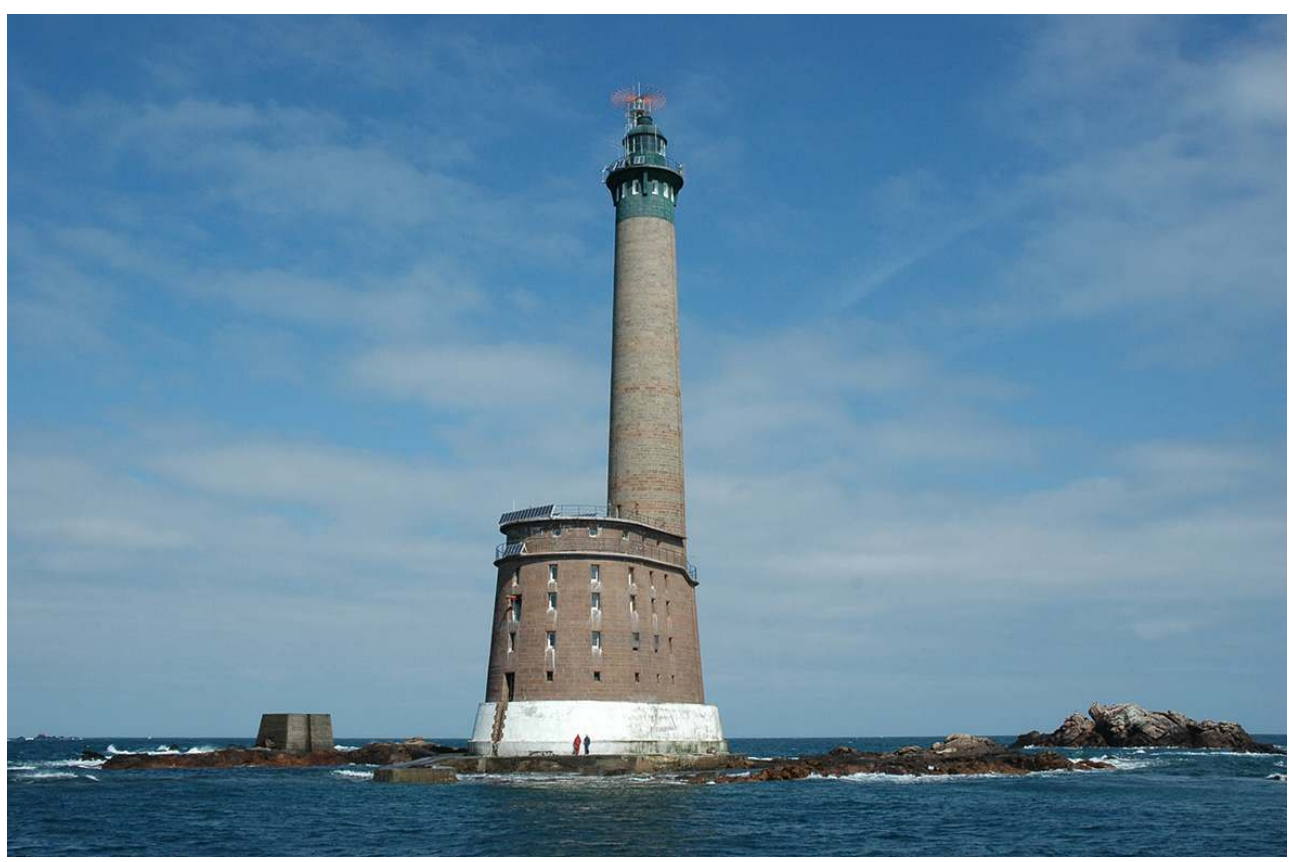

Phare des Roches-Douvres (Côtes d'Armor), architectes Henry Auffret et Joël Hardion, 1954. II est inscrit au titre des monuments historiques depuis le 31/12/2015 et en cours de classement (avis favorable de la CNMH du 15 mars 2016).

Phot. Hervé Raulet, 2015. (c) DRAC/CRMH Bretagne.

\section{S'appuyer sur l'actualité patrimoniale ou l'identité forte d'un territoire}

L'État n'est pas toujours la cheville ouvrière du projet, il peut aussi faire naître des dynamiques de protection grâce à l'actualité patrimoniale locale en mettant à profit l'émulation créée autour d'événements locaux : colloques, expositions, commémorations. Ce fut le cas pour le patrimoine de l'École de Nancy, objet dans les années 1990 d'une redécouverte qui culmina avec la commémoration « Nancy $1900 »$ en 1999. La plupart des édifices Art nouveau de Nancy ont été protégés durant cette décennie. Plus récemment, l'exposition « 1945-1975 en Meurthe-et-Moselle : églises en quête de modernité » réalisée par le service territorial de l'Architecture et du Patrimoine et l'association des Archives modernes de l'architecture lorraine (AMAL) en 2012, présentée à la Cité de l'architecture et du patrimoine du 11 décembre 2013 au 31 janvier 2014, a attiré l'attention sur les églises de la période des Trente Glorieuses restées jusqu'ici relativement méconnues. Cette initiative, qui a associé les décideurs politiques, a abouti à la labellisation "Patrimoine $\mathrm{du} \mathrm{xx}^{\mathrm{e}}$ siècle» de l'ensemble des églises présentées, à l'inscription, le 10 février 2014, de l'église Saint-Léopold à Lunéville (consacrée en 1954) construite par Paul Jacquot et l'abbé Aubry, et même, le 17 juin 2013, au classement de l'église SaintRémy de Baccarat, construite entre 1954 et 1957 par Nicolas Kazis. À l'heure actuelle, le centenaire de la Première Guerre mondiale et de la première reconstruction voit naître, dans chaque région, une nouvelle émulation annonciatrice de campagnes de protection, en partenariat avec les acteurs locaux. En effet, sur environ 30000 monuments aux morts 
de la guerre de 1914-1918 édifiés en France, une quinzaine seulement étaient protégés au titre des monuments historiques en 2007. Plusieurs CPMH ont engagé des campagnes de repérage puis des sélections en vue de protections. C'est la raison pour laquelle la conservation régionale des Monuments historiques Provence-Alpes-Côte d'Azur a constitué, entre 2007 et 2010, avec l'aide de l'ONAC (Office national des anciens combattants et victimes de guerre) et du Souvenir français, une base de données permettant d'établir une sélection en fonction de différents critères: forme, sujet, originalité, notoriété de l'artiste ${ }^{50} \ldots$

Enfin, les spécificités des territoires, leur économie et leur image touristique sont également des moteurs de la politique patrimoniale. À ce titre, plusieurs régions ont travaillé sur leur patrimoine viticole pour le recenser et le valoriser, afin d'en retenir les éléments les plus intéressants aussi bien sur le plan de l'activité économique que d'une typologie locale à mettre en valeur. Les labels (notamment le label « Patrimoine du $\mathrm{xx}^{\mathrm{e}}$ siècle ») et les protections au titre des monuments historiques, qui répondent à des objectifs différents et complémentaires, sont des outils opérationnels et réglementaires qui sont alors mobilisables. Ainsi, en Languedoc-Roussillon, ce sont entre autres les caves coopératives qui ont retenu l'attention des $\mathrm{CPMH}$ ces dernières années. Elles sont en effet un élément majeur du patrimoine du Languedoc-Roussillon par leur nombre - environ 600 caves construites en un siècle -, l'ampleur des constructions et leur caractère symbolique. L'étude menée en 2008 par l'Inventaire général du patrimoine culturel (conseil régional de Languedoc-Roussillon) et la publication de l'ouvrage Caves coopératives en Languedoc-Roussillon ${ }^{51}$ ont mis en lumière ce patrimoine exceptionnel.

Le groupe de travail de la direction régionale des Affaires culturelles sur le Label « Patrimoine du $\mathrm{Xx}^{\mathrm{e}}$ siècle » a utilisé cette étude pour sélectionner dix caves pour le Gard, dont deux (Tavel et Saint-Théodorit) ont été proposées pour une inscription au titre des monuments historiques. Toutes, sauf celle de Saint-Christol-lès-Alès, due à Louis Pierredon, sont l'œuvre d'Henri Floutier (1896-1973), architecte qui a occupé une place majeure dans le Gard, réalisant près de cinquante caves et de nombreux bâtiments publics. La commission régionale du patrimoine et des sites (CRPS) du 14 février 2013 a confirmé ce choix. La cave de Tavel est toujours en activité et l'arrêté d'inscription signé par le préfet de région le 11 juin 2013, en accord avec les coopérateurs, s'étend à la totalité de la partie historique construite en 1937 ainsi qu'aux façades et toitures des premiers agrandissements. La plaque du label « Patrimoine du $\mathrm{xx}^{\mathrm{e}}$ siècle » a été remise le 23 juillet 2013 (fig. 16). 


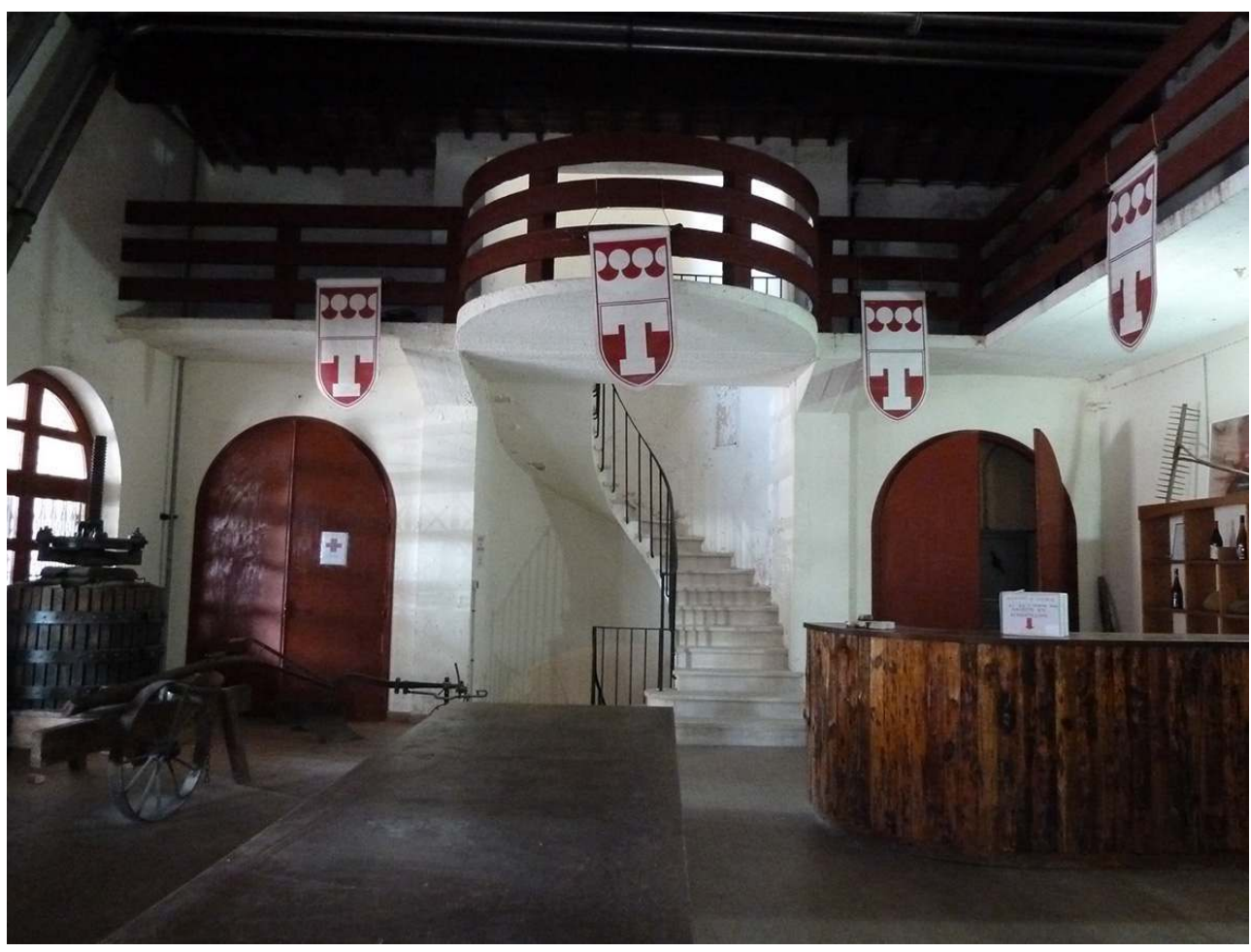

Cave coopérative de Tavel.

Phot. Josette Clier, 2016. (C) DRAC/CRMH LRMP.

\section{Valoriser son action au sein des DRAC}

En aval de la procédure de protection, après la publication de la décision, c'est au CPMH qu'échoient l'organisation et la gestion de la documentation réunie. Considéré comme une archive courante, le dossier requiert son classement physique au sein du service des Monuments historiques, ainsi que l'envoi d'une copie à la médiathèque de l'Architecture et du Patrimoine. Grâce à ces versements, fiches sur les monuments et leurs photos sont mises en ligne dans les bases de données « Architecture et Patrimoine » du ministère de la Cultur $^{\mathrm{r}} \mathrm{e}^{52}$. Le CPMH assure une veille documentaire sur les monuments historiques de son territoire pour alimenter régulièrement les dossiers et répond aux sollicitations multiples des partenaires souhaitant y avoir accès (maîtres d'œuvre, collègues instruisant le contrôle scientifique et technique (CST), chercheurs, étudiants, etc.). Il assure aussi ponctuellement des présentations du métier et du domaine auprès de publics scolaires ou professionnels. À cet égard, le travail engagé préalablement à l'inscription de la Cité paysanne de Marckolsheim (Bas-Rhin) est exemplaire. Sélectionné par un groupe de travail réunissant des experts français et allemands autour de la thématique du "patrimoine de la reconstruction de 1940-1944 en Alsace », cet ensemble de 14 fermes modèles construit entre 1940 et 1942 a été inscrit par arrêté du 24 octobre 2012. Le dossier documentaire a réuni des chercheurs en histoire de l'architecture, en histoire de l'art, en histoire régionale, en ethnologie, des paysagistes, des architectes, etc. Cette opération a donné lieu à des communications destinées à un public varié, à une journée d'étude thématique à Strasbourg, en octobre 2010, à une participation au colloque organisé sur ce sujet en $2013^{53}$ et à la publication d'un article dans la revue In Situ ${ }^{54}$. 
Par les présentations synthétiques qu'il prépare à l'intention des membres de la commission, le CPMH se fait également « vulgarisateur » du patrimoine. En effet, à l'issue d'une recherche de plusieurs mois, il doit faire comprendre l'intérêt d'un édifice en quelques minutes à des personnes qui ne le connaissent souvent pas du tout. Cet effort de synthèse et de pédagogie est essentiel dans le travail du chargé de la protection. L'accueil des consultants extérieurs et la communication des dossiers de protection par le CPMH sont aussi le moyen d'assurer une transmission des connaissances. De par sa connaissance des monuments, le CPMH est également régulièrement sollicité dans le cadre de manifestations nationales comme les Journées européennes du patrimoine ou les Rendezvous au jardin, quand il n'est pas lui-même le correspondant chargé de leur organisation. On fait également appel à lui lors de la pose de plaques «monument historique » pour venir présenter l'édifice sous forme de visite guidée ou de conférence. Lorsqu'il en a le temps et les moyens, le CPMH peut être amené à développer des outils de valorisation et de communication. La collection « Patrimoine restauré », par exemple, qui a pour but de faire connaître l'action des CRMH, publie souvent ses contributions. Des initiatives des DRAC sont aussi à souligner, comme la collection "Duo » et la collection "Chantiers ", créées par la DRAC Languedoc-Roussillon pour faire découvrir des chantiers de restauration, des édifices labellisés « Patrimoine $\mathrm{du} \mathrm{xx}^{\mathrm{e}}$ siècle » ou encore des immeubles et objets d'art protégés au titre des monuments historiques. Dans les différents volumes de cette collection, on trouve souvent des CPMH parmi les auteurs, aux côtés de chercheurs et professionnels extérieurs à la DRAC, bel exemple de collaboration renforcée autour de la valorisation du patrimoine et de la transmission du savoir (fig. 17).

Figure 17
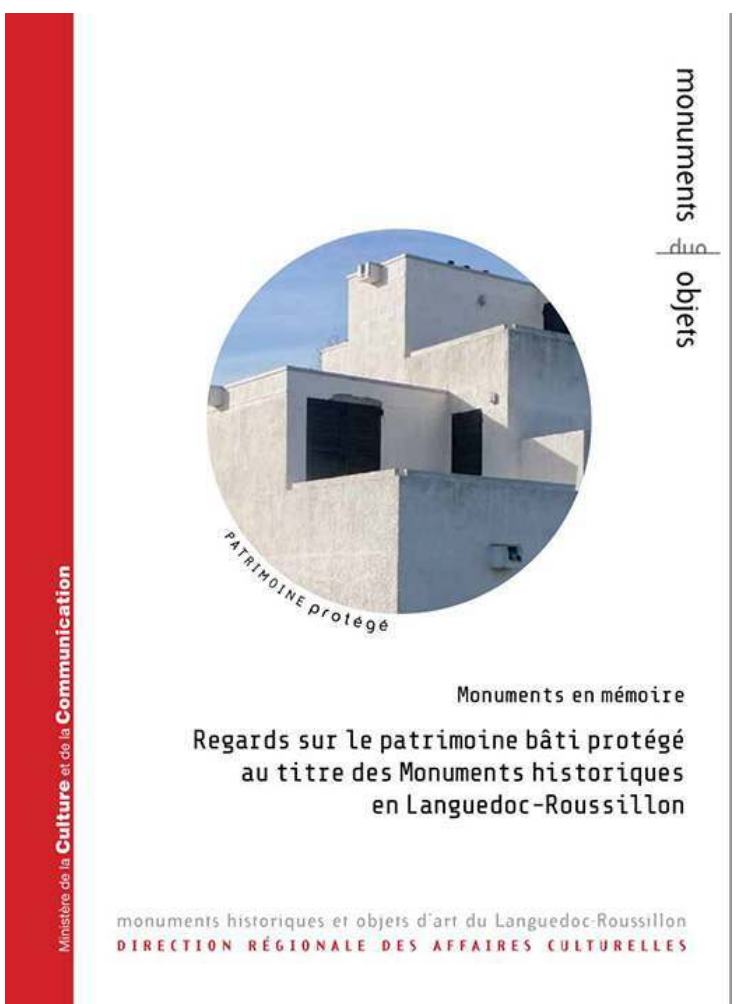

Regards sur le patrimoine bâti protégé au titre des Monuments historiques en Languedoc-Roussillon

Couverture de l'édition de la collection DUO Patrimoine protégé, intitulée Monuments en mémoire. Regards sur le patrimoine bâti protégé au titre des monuments historiques, 2014.

(c) DRAC LRMP. 


\section{Un métier en pleine mutation}

\section{Le rôle du CPMH dans l'évolution de la notion de patrimoine}

L'évolution des champs d'étude du CPMH a suivi celle de l'histoire de l'art, depuis une histoire des formes et du style vers une histoire sociale, économique... de l'art. Il ne s'agit plus seulement pour le CPMH de replacer l'édifice dans l'histoire des styles mais de rechercher son intérêt en tant que témoin des mutations de la société. La notion de monument historique s'est elle aussi profondément transformée: elle recouvre aujourd'hui des réalités monumentales qui vont de la préhistoire au $\mathrm{xx}^{\mathrm{e}}$ siècle et prend en compte de nouvelles catégories d'édifices appartenant au patrimoine industriel, rural, commercial. Puits de mines, forges, jardins, immeubles et villas de banlieue, fontaines de villages et fermes isolées sont autant de nouveaux monuments historiques à découvrir et à comprendre ${ }^{55}$.

Le cas particulier du Fort Chabrol à Épernay (Marne) est symptomatique de la prise en compte de ces nouvelles catégories de patrimoine : lieu de production de plants de vigne pour régénérer le vignoble champenois après l'attaque du phylloxéra, c'est un bâtiment unique en France qui était en fonction au début du $\mathrm{xx}^{\mathrm{e}}$ siècle. L'établissement a permis de renouveler complètement le vignoble Moët et Chandon en une trentaine d'années et a servi de lieu d'apprentissage technique pour les vignerons de Champagne confrontés aux dommages causés par le phylloxéra. Sa disposition correspond aux phases de production, en nombre, des greffes. Il a été inscrit au titre des monuments historiques le 6 décembre 2012 (fig. 18).

Figure 18

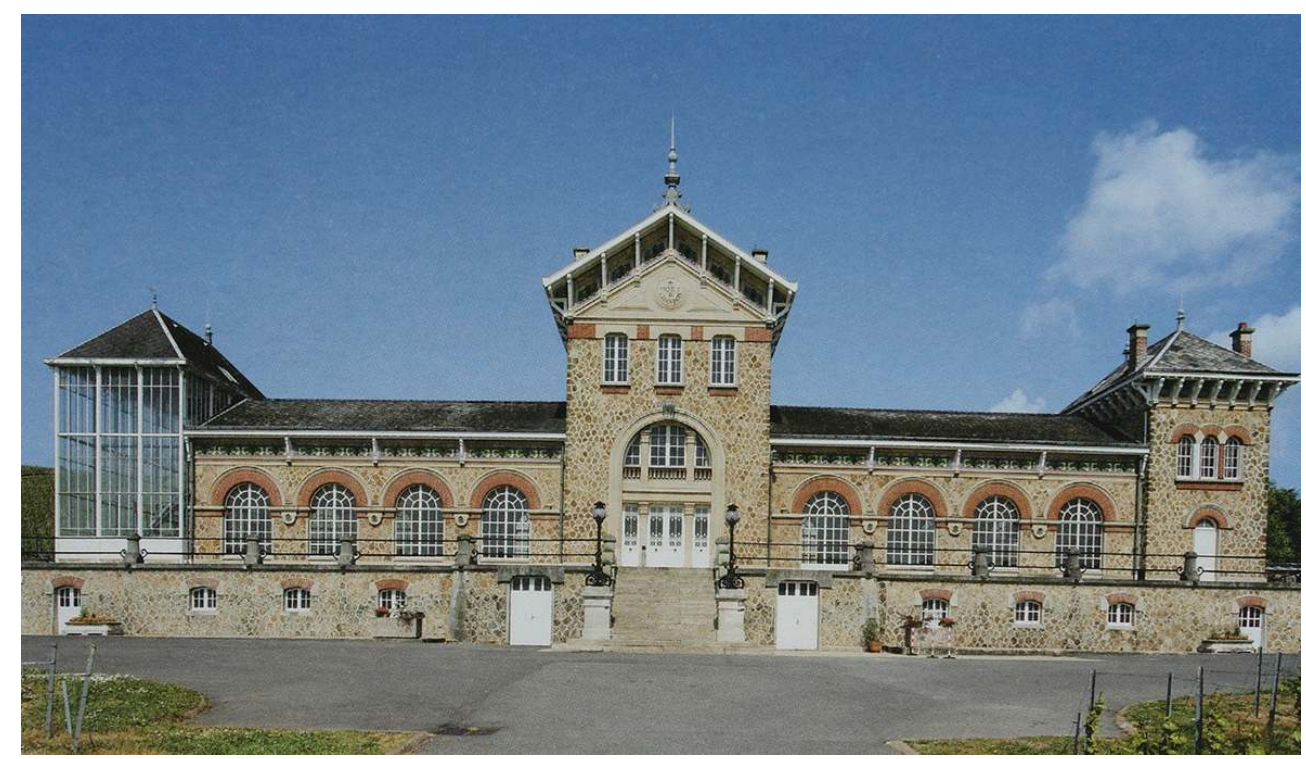

Épernay, le fort Chabrol (ancienne école de viticulture).

Phot. Gilles Vilain, 2012. (c) DRAC/CRMH Grand Est. 


\section{L'évolution de la politique de protection du patrimoine}

Le chargé de la protection au titre des monuments historiques contribue à la définition, à la mise en œuvre et au suivi de la politique de protection régionale; dans ce domaine, l'évolution de la politique a contribué à professionnaliser les métiers de la protection. En effet, des premières listes élaborées à partir d'un repérage rapide des éléments phares du patrimoine national à une politique plus incitative et plus raisonnée, par le recensement et la sélection, sans oublier la prise en compte individuelle des demandes de la société, ce processus a justifié le développement d'une méthodologie exigeant un travail par thématiques et la définition de critères. Une instruction du directeur de l'Architecture et du Patrimoine du $1^{\text {er }}$ décembre 2008 a précisé les orientations sur les critères de la protection des immeubles au titre des monuments historiques. Ceux-ci sont particulièrement déterminants quand les commissions (DP, CRPS et CNMH) sont confrontées à une typologie d'édifice en série ou existant en grand nombre sur le territoire. En effet, les éléments les plus emblématiques du patrimoine ayant été pour la plupart protégés, les nouveaux éléments patrimoniaux pris en compte relèvent souvent plus de la série ou de patrimoines plus modestes (architecture rurale, industrielle...) dont la sélection et la contextualisation doivent être solidement étayées. Citons le cas des «préfabriqués à parois inclinées » Fillod de Gueugnon (Saône-et-Loire) : le CPMH a mis l'accent sur le caractère innovant de ces pavillons et leur lien fort avec l'histoire industrielle et sociale du lieu; ils sont les témoins de l'aventure industrielle d'un visionnaire pionnier dans le principe de la préfabrication, et aussi de l'aventure industrielle des forges de Gueugnon, fleuron de la ville. Ils possèdent aussi une dimension historique pour être liés aux mouvements de populations découlant de l'immigration et de la guerre, hébergements d'urgence pour les ouvriers italiens et les prisonniers de guerre allemands. Ce sont les seuls exemples de hangars protégés au titre des monuments historiques en France. Lors de l'examen en commission s'est posé également le problème du choix des éléments à protéger parmi les nombreux exemples conservés sur le territoire de la commune ainsi que celui du statut de ces éléments modulables, déplaçables et conçus comme des habitations provisoires (fig. 19). Finalement, cinq pavillons Fillod ont été inscrits, le 26 août 2015, sur les critères de l'authenticité et de la mémoire de la guerre puisqu'ils avaient servi de prison. 


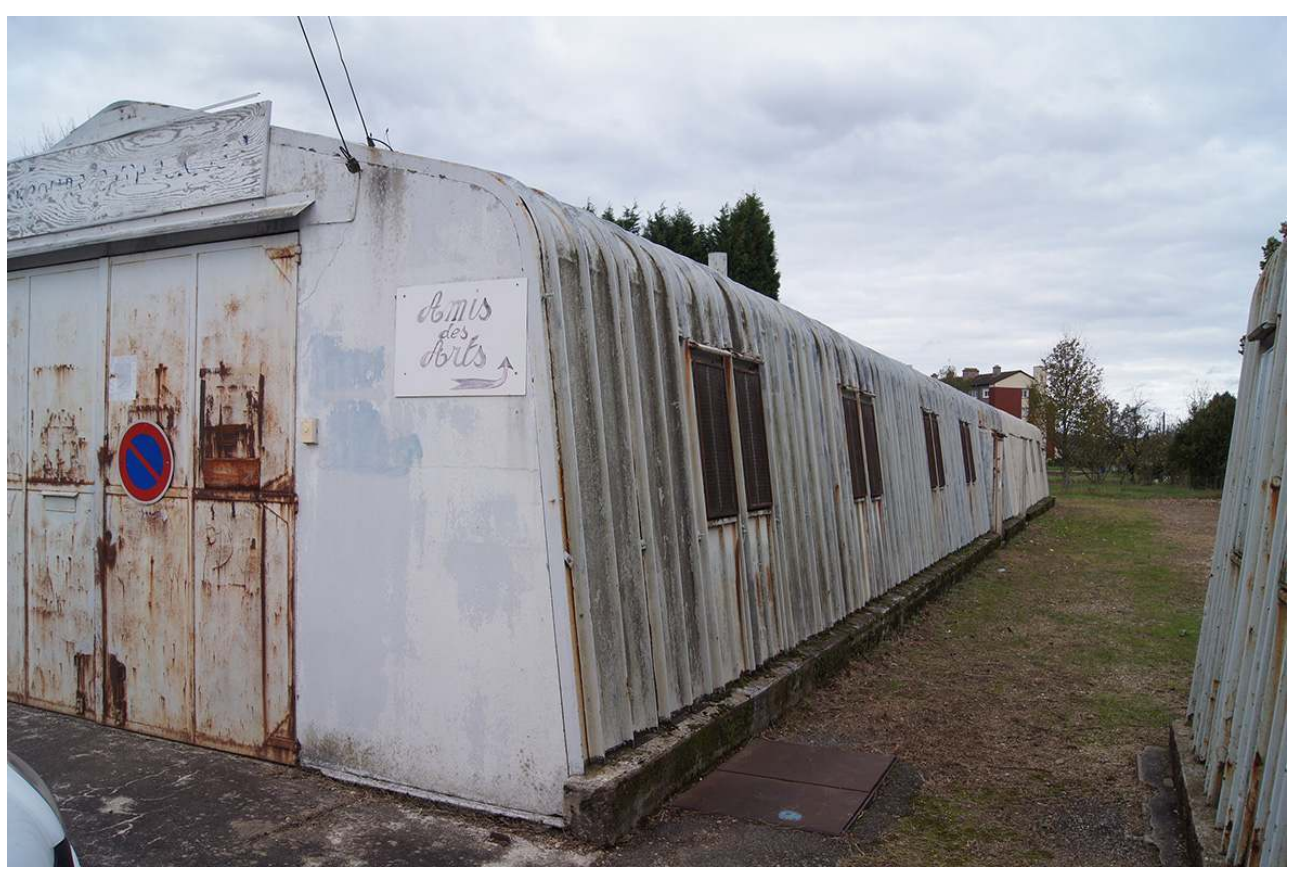

Gueugnon, pavillon Fillod.

Phot. Sophie Loppinet Méo, 2013. @ DRAC/CRMH Bourgogne - Franche-Comté.

\section{L'apparition de nouveaux champs patrimoniaux}

61 L'élargissement de la notion de patrimoine implique le développement continu des connaissances du CPMH. Si l'action de propriétaires passionnés ou d'associations motivées mais aussi l'initiative du service lui-même, avec l'engagement de thématiques, joue souvent un rôle déterminant, le recenseur lui-même peut être force de proposition pour mettre en valeur de nouveaux types de patrimoine et convaincre les propriétaires et les acteurs concernés.

L'exemple le plus emblématique de l'élargissement des champs patrimoniaux est certainement celui du patrimoine industriel. Pour ce patrimoine dont l'intérêt social le dispute souvent à l'intérêt scientifique et technique, les critères de protection ont évolué : au départ, la notion de patrimoine scientifique et technique a primé dans les instances officielles, malgré une demande sociale liée au devoir de mémoire. Ce qui a conduit à des protections partielles, sous forme de totems (chevalement, machines mais pas équipements sociaux). La tendance actuelle est de prendre en compte la dimension sociale de ce patrimoine avec des protections complémentaires pour les lieux de vie. La protection progressive des témoignages du bassin minier et sidérurgique, important, du Creusot-Montceau-les-Mines, en voie de disparition, en est une bonne illustration. Les usines Schneider et le lavoir à charbon ont d'abord été protégés au titre des monuments historiques, respectivement en 1975 et 2000, avant que soit reconnu l'intérêt historique de la cité ouvrière de la Combe des mineurs (IMH en 1980), puis la maison des syndicats et le dispensaire d'hygiène sociale (2012), témoins d'une activité industrielle florissante et d'une organisation sociale prégnante au sein de la commune (fig. 20). 


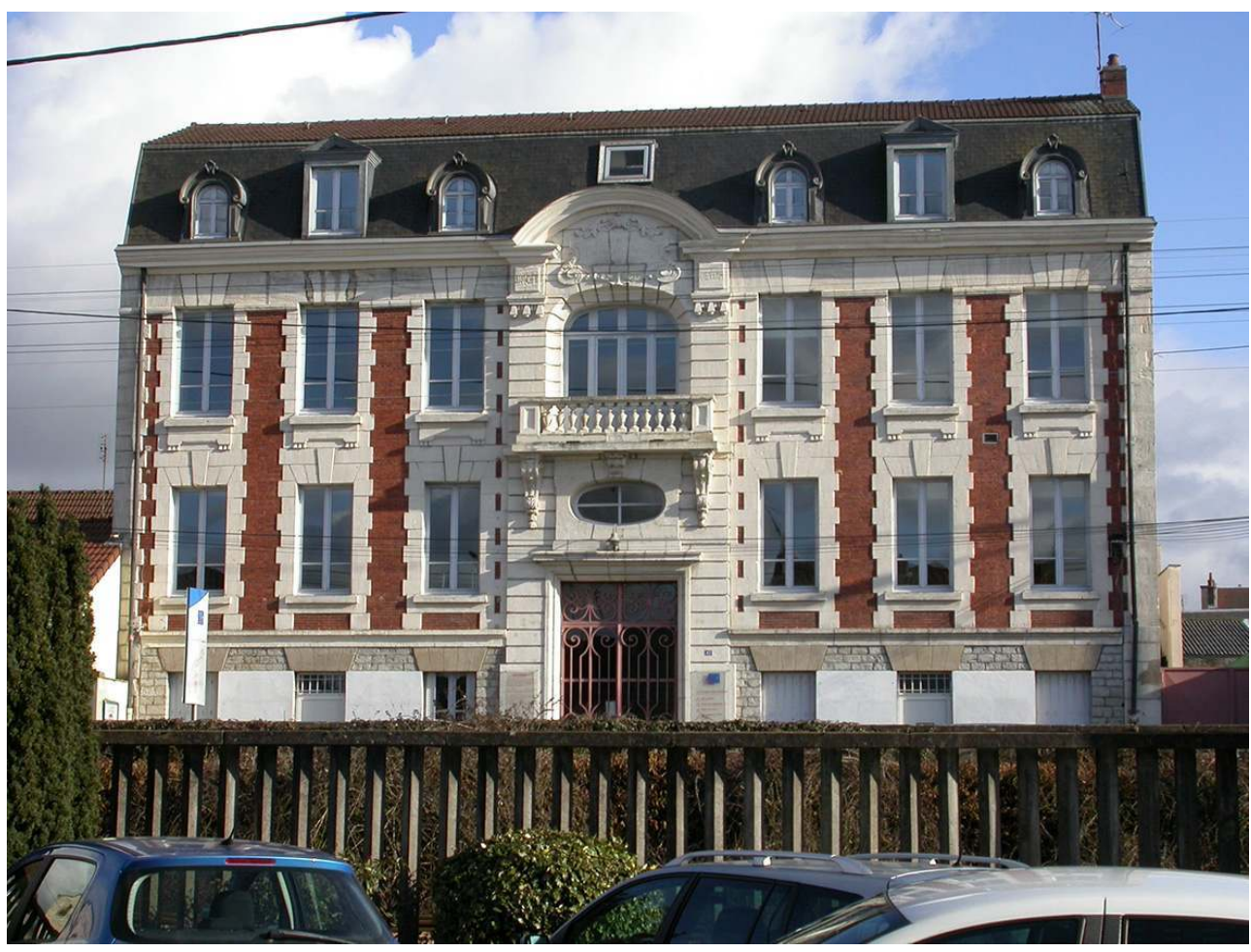

Montceau-les-Mines : maison des syndicats.

Phot. Sophie Loppinet Méo, 2012. @ DRAC/CRMH Bourgogne - Franche-Comté

63 La protection au titre des monuments historiques de réclames sur un pignon à Paris, au 10 rue des Martyrs, illustre l'élargissement de la notion de patrimoine à des éléments du quotidien. Ces réclames ont été découvertes fortuitement, la cabane en bois qui les masquait ayant préservé toute leur fraîcheur. Ces réclames sont datées de 1908 et signées des peintres Defoly et Vavasseur, éléments qui ont conduit à envisager leur protection. Le caractère exceptionnel de cette découverte a été souligné, ainsi que le fait que notre époque ait interdit ce type de publicité murale. Ce sont aujourd'hui les seules réclames protégées au titre des $\mathrm{MH}$ en France ${ }^{56}$.

Le CPMH se trouve ainsi de plus en plus souvent confronté à des patrimoines inédits, jusqu'à des lieux du quotidien. Ainsi, lorsque le dernier exemplaire bien conservé des quatre supermarchés dessinés par Claude Parent à Sens a été menacé de dénaturation, le groupe de travail régional du label « Patrimoine du $\mathrm{Xx}^{\mathrm{e}}$ siècle » de la DRAC de Bourgogne, auquel participent les $\mathrm{CPMH}$, et des personnalités de l'architecture ont conjugué leurs efforts pour faire protéger l'édifice au titre des monuments historiques. Cette initiative a été soutenue par une pétition de citoyens. Le dossier de protection a fait la synthèse des critères justifiant la protection, soit la notoriété de l'auteur, l'intérêt de l'édifice eu égard à l'histoire (implantation en France des premiers supermarchés d'après le modèle américain), son intérêt pour l'architecture contemporaine (en particulier la théorie de la fonction oblique exprimée dans la galerie à pans inclinés) et son intégrité en comparaison des autres centres commerciaux dessinés par Claude Parent ${ }^{57}$. Le bâtiment a été finalement inscrit au titre des monuments historiques en totalité par arrêté du 10 juin 2011. À l'initiative du CPMH, de nombreux échanges sur les possibles étendues de la protection au titre des monuments historiques et d'un périmètre de protection des 
abords ont eu lieu avant la présentation en commission, à laquelle des représentants du groupe ont assisté (fig. 21).

Figure 21

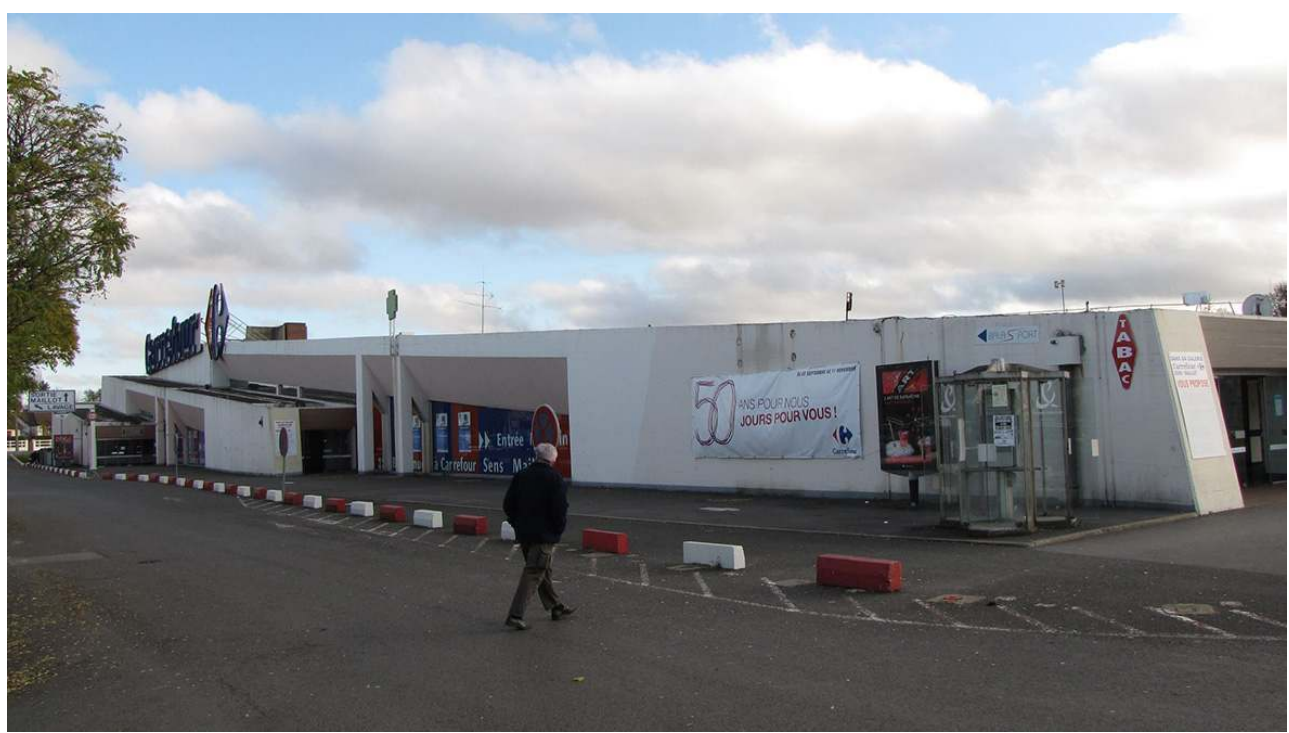

Supermarché dessiné par Claude Parent à Sens.

Phot. Viviane Rat-Morris, 2011. (c) DRAC/CRMH Bourgogne - Franche-Comté.

Le chargé de protection voit aussi son domaine d'étude s'élargir considérablement avec la prise en compte d'un territoire dans toutes ses spécificités. Citons ici l'exemple de la cité expérimentale de Merlan à Noisy-le-Sec (Seine-Saint-Denis), sorte de show-room urbain de la nouvelle architecture de maisons individuelles, qui a fait l'objet d'un arrêté global, le 28 décembre 2000. Celui-ci prend en compte l'ensemble des maisons construites pour reloger la population sinistrée de la dernière guerre, ainsi que la voirie. La cité fut inaugurée en juillet 1946 ; elle comprenait initialement 56 pavillons dont 43 sont toujours en place. La propriété de l'ensemble se partage entre privé, État, commune et association 58 .

\section{La création de nouvelles formes de protections : les labels}

L'émergence de ces nouveaux champs patrimoniaux que le ministère a souhaité porter et mettre en valeur s'est manifestée également par la création de labels ${ }^{59}$. Ces derniers ont en quelque sorte remplacé un troisième niveau de protection dont le ministère a souvent débattu sans le concrétiser juridiquement. Thématiques, typologiques ou chronologiques, sans impact juridique, leur attribution est aujourd'hui un moyen de valoriser et de sensibiliser, indépendamment ou avant une mesure de protection. Des groupes de travail ont donc souvent été instaurés pour sélectionner les éléments les plus pertinents, et les CPMH assument la plupart du temps la gestion de ces labels, compléments logiques de leur démarche patrimoniale ${ }^{60}$.

Les groupes de travail sur le patrimoine du $\mathrm{xx}^{\mathrm{e}}$ siècle en sont l'illustration. Composés de spécialistes du domaine et de fonctionnaires, ils ont examiné dans plusieurs régions l'intérêt d'un patrimoine quelquefois très récent et à ce titre, parfois difficilement perçu comme tel, attribuant un label « Patrimoine du $\mathrm{xx}^{\mathrm{e}}$ siècle » aux édifices témoignant d'une 
étape dans l'évolution de l'architecture contemporaine et se prononçant en faveur d'une protection pour les plus insignes ${ }^{61}$.

68 Le label «Jardin remarquable » implique, lui, non seulement la constitution de dossiers mais aussi une évaluation selon les critères définis par la circulaire $n^{\circ} 2008 / 011 d u$ 29 octobre 2008. Il est mis en œuvre par un groupe de travail expert désigné par le préfet de région. Ainsi, est considéré l'intérêt patrimonial mais également botanique des jardins, et surtout l'existence d'un véritable plan de gestion garantissant la pérennité de ce patrimoine vivant, critère essentiel pour la labellisation. En ajoutant à sa mission celle de correspondant du label « Jardin remarquable », le CPMH élargit son champ d'action et son expertise. Il encourage ainsi une politique de plan de gestion des jardins historiques, essentielle au contrôle scientifique et technique de ces monuments historiques pas comme les autres. Ainsi, si les critères diffèrent, il y a bien une complémentarité entre label et protection pour les jardins. Le parc de la Marseillaise à Guebwiller (Haut-Rhin) a ainsi reçu le label «Jardin remarquable» en 2006 avant d'être inscrit au titre des monuments historiques, le 19 août 2013 (fig. 22).

Figure 22

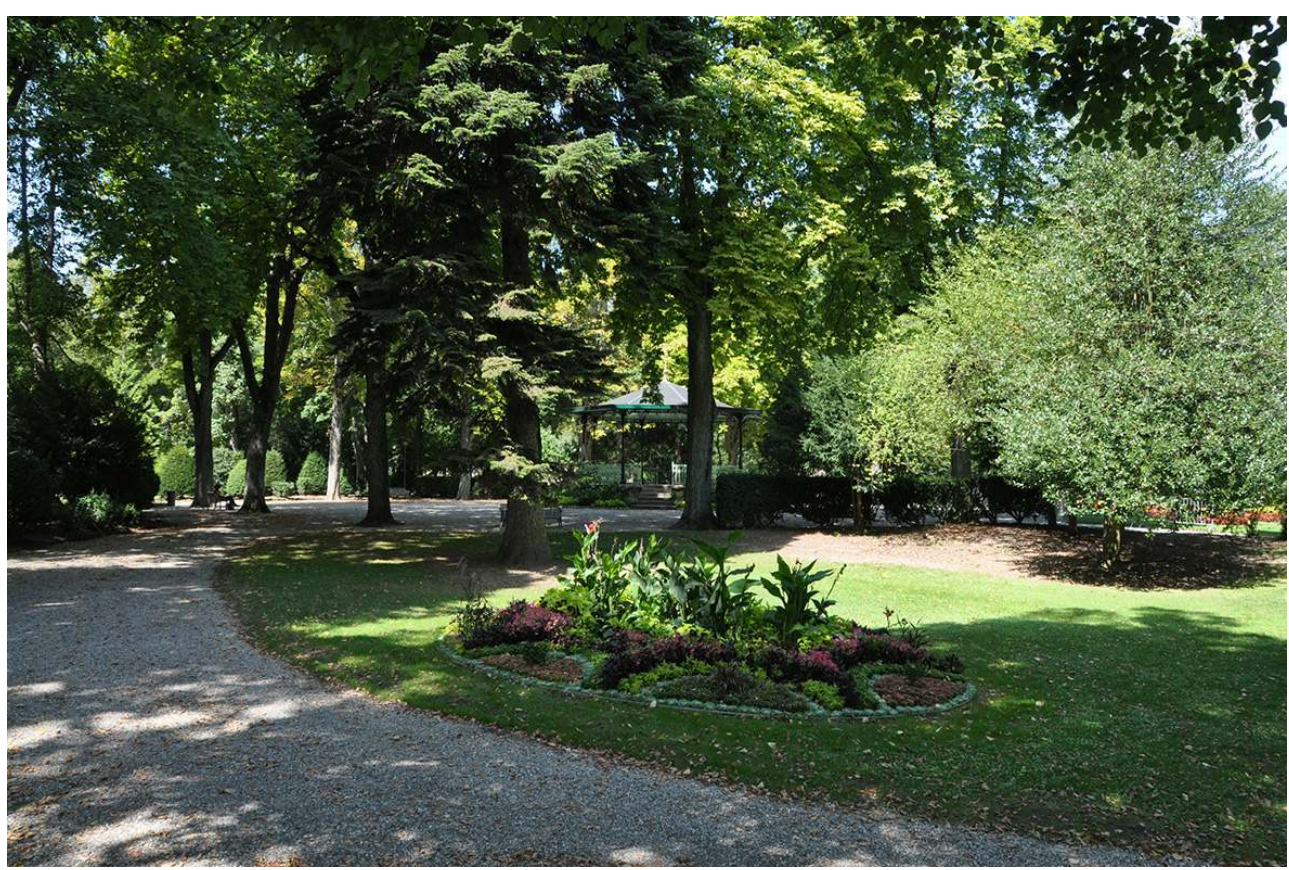

Le Parc de la Marseillaise à Guebwiller.

Phot. Clémentine Albertoni, 2011. (c) DRAC/CRMH Grand Est.

69 Nombreux sont les exemples qui illustrent l'approche différenciée entre labels et protection, et l'ambiguïté administrative de l'aboutissement de certains dossiers, comme celui du cinéma expérimental Panrama de Philippe Jaulmes à Clapiers (Hérault). Présenté du bout des lèvres en délégation permanente de la CRPS, puis pour le label « Patrimoine du $\mathrm{XX}^{\mathrm{e}}$ siècle " par le groupe de travail, il a été inscrit après avis favorable de la CRPS, et son classement est aujourd'hui proposé! (fig. 23). On voit combien le CPMH s'investit dans toutes les étapes de la patrimonialisation, jusqu'à des actions quasi militantes, en particulier pour défendre le patrimoine architectural contemporain à l'égard duquel il a un vrai rôle pédagogique. Sur ce plan, l'association «MoHiCan », qui regroupe les CPMH au sein de l'Association des historiens de l'architecture, n'est pas inactive. 
Figure 23

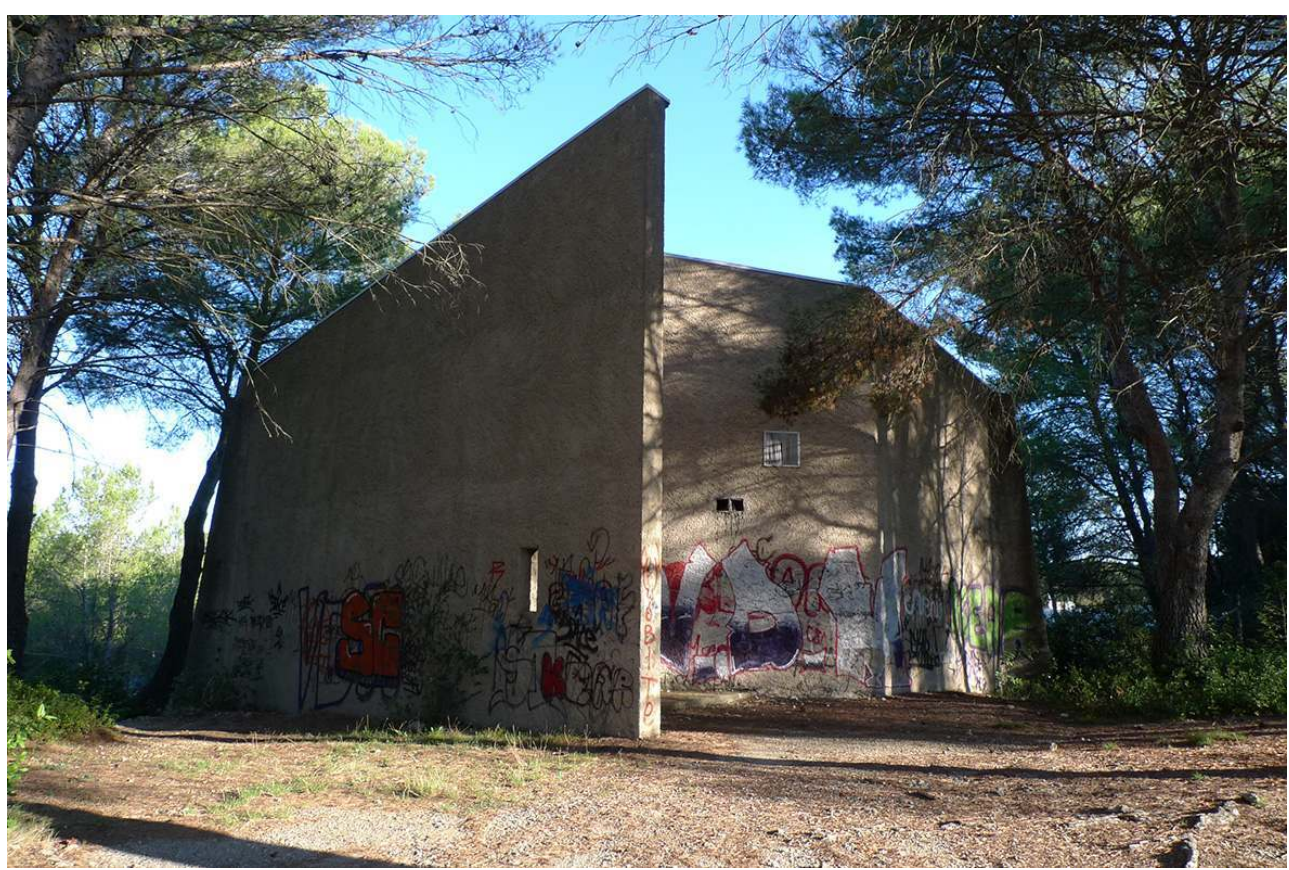

Cinéma Panrama à Clapiers : vue générale du côté de l'entrée du public : état actuel.

Phot. Yvon Comte, 2011. (c) DRAC/CRMH LRMP.

\section{De nouvelles compétences, de nouveaux outils}

À l'origine centrées sur la préparation des dossiers de protection et l'acte juridique qui en découle, la gestion de la documentation produite, les tâches des CPMH se sont beaucoup diversifiées, suivant en cela la volonté ministérielle de transversalité des fonctions. L'apparition de nouvelles missions exige de la part de ce professionnel l'adaptation permanente de ses compétences. Le développement des outils numériques et la complexification des aspects juridiques du métier ont généré en contre-partie des exigences et des attentes plus grandes, surtout pour ce qui touche à la valorisation de l'information.

\section{Les nouvelles technologies : une exigence nouvelle}

L'exigence croissante de mise à disposition des données issues de la protection à destination d'un public large a amené le ministère à intensifier le traitement informatisé des données et à créer des bases de données en ligne pour donner libre accès à l'information et faciliter la gestion des travaux en aval. Le CPMH est chargé de l'alimentation de ces bases de traitement et de gestion de la documentation: base de gestion AgrEgée, bases photos internes aux services SIG...

72 Le passage au numérique a nécessité une importante mise à jour des données afin d'introduire une hiérarchie dans la description des édifices. La présentation des ensembles ou bâtiments en parties d'édifices cohérentes est devenue nécessaire pour simplifier le traitement informatisé des données des campagnes de travaux futures. Cela suppose que l'on possède une connaissance approfondie du bâtiment, grâce à l'étude qui 
en est faite dans le cadre du dossier de protection, et que l'on anticipe l'intervention des corps de métiers.

Le géo-référencement des données patrimoniales, tels l'Atlas du patrimoine ${ }^{62}$ ou le géoportail ${ }^{63}$, a nécessité en outre une définition précise de l'étendue de la protection, transcrite visuellement par le dessin et a fait naître des projets de campagnes de révision de protection pour consolider juridiquement les arrêtés de protection.

Parallèlement s'engage au sein du ministère une démarche de présentation dématérialisée du dossier de protection pour sa mise à disposition à la médiathèque de l'Architecture et du Patrimoine. Les dossiers des régions Languedoc et Poitou ont été numérisés; ils sont reliés à la notice Mérimée, domaine $\mathrm{MH}$, sous l'intitulé « dossier de protection $»^{64}$.

75 L'arrivée du numérique a multiplié les possibilités d'information, notamment pour la photo qui permet de documenter davantage les édifices, ce qui va de pair avec la nécessité de consolider les actes juridiques sur le fond dans le cadre du dossier de protection et de sa présentation en commissions.

\section{Transversalité des fonctions}

76 La participation désormais effective du CPMH aux réunions du contrôle scientifique et technique (CST) ou de suivi des travaux sur monument historique, soumis à l'avis de la CRMH, porte sur la précision de l'étendue de la protection et la définition de la valeur patrimoniale (ce qui a motivé la protection) : il donne un avis sur les projets de travaux sur les monuments historiques de la région, en collaboration avec les autres agents de la CRMH ; il participe à l'élaboration des portés à connaissance et cahiers des charges en vue des travaux, à l'élaboration d'études, de plans de gestion, à l'établissement d'un état sanitaire périodique des monuments historiques. De nombreux débats ont eu lieu, par exemple, à propos de la restauration de peintures murales impliquant un choix sur la période de décor à mettre en valeur. La confrontation des sondages des restaurateurs avec l'analyse du bâti et la prise en compte de l'histoire de l'édifice permet de dégager un parti pris de restauration harmonisant décor et architecture.

\section{Conclusion}

77 Le chargé de la protection des monuments historiques voit actuellement ses responsabilités s'élargir, dans le sens d'une participation à toutes les étapes de la patrimonialisation: inventaire thématique, étude monographique, participation au contrôle scientifique et technique, vulgarisation auprès des publics, archivage numérique et dématérialisation de la documentation. Ces professionnels doivent toutefois rester vigilants car cette mutation se fait dans le contexte de la fusion des régions, à laquelle il faut s'adapter, et de raréfaction des postes. Face au manque de personnel et aux changements administratifs, il pourrait être tentant d'externaliser la constitution des dossiers de protection, faute de temps à consacrer à la recherche, à l'analyse et à la rédaction. Cette externalisation supprimerait pourtant la complémentarité entre l'approche scientifique et l'ancrage dans le terrain, qui s'enrichissent mutuellement et font la force et l'intérêt du métier. Car c'est justement par sa perception globale du territoire, par sa connaissance des enjeux et des acteurs que le chargé de la protection, en tant que fonctionnaire au regard impartial, peut apporter un avis éclairé sur une 
proposition de protection, ce que ne pourrait pas faire un prestataire extérieur recruté pour une mission précise de recherche en temps limité. Le CPMH risquerait de s'éloigner du territoire dont il a la charge, voire de sa mission première, la protection elle-même. Pourtant, en contradiction avec un discours ambiant et déjà ancien qui voudrait que la démarche de protection des monuments historiques ait un terme et qu'il n'y ait bientôt "plus rien à protéger", la vitalité de la notion de patrimoine rend la mission de protection du CPMH toujours plus actuelle. Depuis 2000, 59 classements et 338 inscriptions sont prononcés en moyenne chaque année, huit séances de la CNMH sont tenues ainsi qu'une cinquantaine de CRPS. En 2014, 520 nouvelles demandes ont été formulées ${ }^{65}$. Ces chiffres démontrent la réalité de la protection des monuments historiques qui constitue, autant que la restauration et l'entretien, le quotidien des services patrimoniaux.

Engagé sur son territoire, le chargé de la protection des monuments historiques exerce un métier qui offre une richesse d'actions et de rencontres, ce qui compense la frustration de ne pouvoir tout protéger et tout étudier. Face aux bouleversements de la nouvelle organisation territoriale et aux dotations en baisse, il veille, avec le soutien de l'association MoHiCan et de son réseau d'interlocuteurs, à continuer de faire rayonner le patrimoine, dans toute sa diversité.

\section{BIBLIOGRAPHIE}

JAMOT, Francis, MARX, Jean, AUDIBERT, Martine, DENANTE, Sylvie. La protection des immeubles au titre des monuments historiques : manuel méthodologique. Paris : ministère de la Culture et de la Communication, direction de l'Architecture et du Patrimoine, 2003.

Association des personnels de documentation des affaires culturelles. Levez le voile sur un métier du patrimoine : recenseur documentaliste chargé de la protection des MH. Oct. 1994.

Les monuments historiques demain. Colloque organisé par la direction du Patrimoine, Paris, La Salpêtrière, novembre 1984. Paris : ministère de la Culture et de la Communication, 1987.

AUDUC, Arlette. Quand les monuments construisaient la Nation. Le service des monuments historiques de 1830 à 1940. Paris : Comité d'histoire du ministère de la Culture, 2008.

BERCÉ, Françoise. Des monuments historiques au patrimoine, du XVIII siècle à nos jours, ou «Les égarements du cour et de l'esprit ». Paris : Flammarion, 2000.

FLOUQUET, Sophie. « Profession : chargé d'études documentaires ». Journal des Arts, n 303, 15-28 mai 2009.

FRANCOIS, Michèle. « Roger Hyvert (1901-1988), recenseur des monuments historiques de 1945 à 1965 ». Bulletin de la Société d'études scientifiques de l'Aude, t. CXIV, 2014, p. 159-169. <http:// www.sesa-aude.fr/Bulletin-de-2014-Tome-CXIV>

GALLO, Emmanuelle. « À quoi servent (encore) les historiens de l'architecture ? ». Le Moniteur, 11 février 2005. 
GOURBIN, Patrice. Les monuments historiques de 1940 à 1959. Administration, architecture, urbanisme. Rennes : Presses universitaires de Rennes, 2008.

LAURENT, Xavier. Grandeur et misère du patrimoine, d'André Malraux à Jacques Duhamel, 1959-1973. Paris : École nationale des chartes/Comité d'histoire du ministère de la Culture, 2003.

LEFORT, Nicolas. Patrimoine régional, administration nationale : la conservation des monuments historiques en Alsace de 1914 à 1964. Thèse d'histoire sous la direction de François Igersheim. Strasbourg : université de Strasbourg, 2013.

MARX, Jean. «Chargés d'études et protection : de la naissance des monuments historiques ». Dans PRIEUR, Michel, AUDRERIE, Dominique. Les monuments historiques, un nouvel enjeu? Actes du colloque international, Limoges, 29-30 octobre 2003. Paris : L'Harmattan, 2004, t. 1, p. 171-192.

RÉAU, Louis. Histoire du vandalisme. Les monuments détruits de l'art français. T. 1. Du haut Moyen Âge au XIX ${ }^{e}$ siècle. T. 2. XIX ${ }^{e}$-XX ${ }^{e}$ siècles. Paris : Hachette, 1959.

Pour aller plus loin :

Pour l'histoire de l'organisation du service des Monuments historiques, voir les ouvrages de Paul Léon (1874-1962), qui fut le principal historiographe du service des Monuments historiques français. Il a notamment été membre de la Commission supérieure des monuments historiques de 1907 à sa mort, soit plus de cinquante ans. Entre autres :

- Les monuments historiques, conservation, restauration. Paris : H. Laurens, 1917.

- La vie des monuments français, destruction restauration. Paris : A. et J. Picard, 1951.

- « La protection des monuments ». La Documentation française illustrée, avril 1952.

- Mérimée et son temps. Paris : PUF, 1962.

- « Les monuments (1939-1955) ». Les monuments historiques de la France. Bulletin des diverses sections de la Commission des monuments historiques..., 1955, p. 3-8.

- « Projets pour la reconstitution de la France ». Les Arts, n 165,1917, p. 11-17.

Les « Entretiens du patrimoine », colloques de la direction du Patrimoine, ont abordé les grandes problématiques techniques et déontologiques des monuments historiques, spécialement « les monuments historiques demain » $\left(n^{\circ} 1\right)$, « les inventaires du patrimoine industriel » $\left(n^{\circ} 2\right)$, « les enjeux du patrimoine architectural du xx siècle » $\left(\mathrm{n}^{\circ} 4\right)$, « de l'utilité du patrimoine » $\left(\mathrm{n}^{\circ}\right.$ $11)$, « meubles et immeubles » $\left(n^{\circ} 13\right)$, « des monuments historiques aux patrimoines : le centenaire de la loi de $1913 »\left(n^{\circ} 17\right)$, « le regard de l'histoire : l'émergence et l'évolution de la notion de patrimoine au cours du xx ${ }^{\mathrm{e}}$ siècle en France » (2001).

La revue Monumental se fait l'écho des expériences de terrain. Le numéro de $2015,1^{\mathrm{er}}$ semestre, a en particulier abordé la question du patrimoine industriel :

SCHOENSTEIN, Frantz. « La protection du patrimoine industriel au titre des monuments historiques 1970-2015», p. 20-23.

LEFEBVRE, Anne et LIARDET, Olivier. « Des monuments historiques au patrimoine mondial, la longue marche du bassin minier du Nord-Pas-de-Calais », p. 40-43.

JUHEL, Enora et MICHARD, Isabelle. « La protection et la gestion urbaine du site de Bataville, Moselle », p. 46-49.

RAT-MORRIS, Viviane. « La protection d'un établissement industriel en activité : l'usine Solvay à Clamecy, Nièvre », p. 50-52. 


\section{NOTES}

1. - Avec la participation des CPMH suivants : Isabelle Baguelin, Hervé Raulet, DRAC Bretagne ; Gilles Vilain, DRAC Grand Est (Châlons-en-Champagne) ; Viviane Rat-Morris, DRAC Bourgogne-Franche-Comté (Dijon) ; Michèle François, DRAC Languedoc-RoussillonMidi-Pyrénées (Montpellier) ; Juliette Pozzo, DRAC Rhône-Alpes-Auvergne (Lyon); Élisabeth Wallez, DRAC Normandie (Rouen); Georges Gonsalvès, DRAC LanguedocRoussillon-Midi-Pyrénées (Toulouse) ; Blandine Jourdan, DRAC PACA ; Josette Clier, Yvon Comte, DRAC Languedoc-Roussillon-Midi-Pyrénées (Montpellier).

2. - La mission des CPMH se limite aux immeubles par nature. Les objets et immeubles par destination sont du ressort des conservateurs des monuments historiques et des conservateurs des antiquités et objets d'arts (CAOA).

3. - MARX, Jean. « Chargés d'études et protection : de la naissance des monuments historiques ». Dans PRIEUR, Michel, AUDRERIE, Dominique. Les monuments historiques, un nouvel enjeu? Actes du colloque international, Limoges, 29-30 octobre 2003. Paris : L'Harmattan, 2004, t. 1, p. 171-192.

4. - Ludovic Vitet (1802-1873) fut nommé premier inspecteur général des Monuments historiques par François Guizot le 25 novembre 1830. Il démissionna le 10 avril 1834. 5. - Prosper Mérimée (1803-1870) devint inspecteur général des monuments historiques en 1834 .

6. - Le statut a été fixé en 1965. Voir infra.

7. - Voir le site : https://inventaire.poitou-charentes.fr/operations/le-patrimoineroman/64-decouvertes/409-art-roman-et-monuments-historiques-en-poitou-charentes [consulté le 22/03/2017].

8. - « Monuments Historiques Culture association nationale ». Créée en 1960, elle réunit les personnels du ministère de la Culture et de la Communication chargés des missions de protection au titre des monuments historiques. Elle compte actuellement une cinquantaine de membres. On se reportera avec intérêt à l'intervention de Jean Marx, président de l'association, au colloque international tenu à Limoges en 2003 (MARX, Jean. Art. cit.).

9. - Créée en 1837, la Commission des monuments historiques effectue un travail d'inventaire, de classement et de répartition des fonds consacrés par l'État à la sauvegarde des monuments jugés intéressants.

10. - Arrêté ministériel du 13 juillet 1944. Créé sous l'Occupation, le service de Recensement des monuments anciens de la France avait pour mission d'établir le Casier archéologique de la France. Son but était d'achever l'inventaire des monuments historiques. Sur ce service, voir GOURBIN, Patrice. Les monuments historiques de 1940 à 1959. Administration, architecture, urbanisme. Rennes : PUR, 2008, p. 127-129.

11. - Instruction sur le recensement des monuments, direction générale de l'Architecture, ministère de la Jeunesse, des Arts et des Lettres (médiathèque de l'Architecture et du Patrimoine, 80/12/1).

12. - Les premiers postes de conservateurs régionaux des Bâtiments de France ont été créés en 1948. Les premières circonscriptions correspondaient au ressort des académies. La création de commissions départementales des monuments historiques était prévue mais ne fut jamais effective. Les conservateurs sont chargés de représenter la direction de 
l'Architecture au niveau local ; la protection reste cependant l'apanage de la Commission des monuments historiques, à Paris.

13. - Le ministre de l'Éducation nationale au ministre des Finances, direction du Budget, 18 septembre 1945 (médiathèque de l'Architecture et du Patrimoine, 80/12/1).

14. - Note pour le directeur des monuments historiques, 17 novembre 1946 (médiathèque de l'Architecture et du Patrimoine, 80/12/1).

15. - La Commission prend le nom de "Commission supérieure des monuments historiques » par décret du 4 avril 1950. La délégation permanente de la première section est chargée de préparer les affaires qui sont soumises à la première section et de donner des avis sur les propositions d'inscription à l'Inventaire supplémentaire des monuments historiques, et sur les questions et les projets de travaux qui ne posent pas de question de doctrine.

16. - Les fiches étaient ensuite centralisées à la direction de l'Architecture. Le Casier archéologique de la France, classé par département, était consultable par les architectes du service en vue des travaux.

17. - Les délégués au recensement avaient été rattachés aux conservations régionales des Bâtiments de France en 1957.

18. - LAPEYRE, André. « Législation et organisation du service des Monuments historiques dans la France métropolitaine au $1^{\mathrm{er}}$ janvier 1967 : aperçu sommaire ». Bulletin monumental , 1967, vol. 125, n 3, p. 279-289. Voir le site: http://www.persee.fr/doc/ bulmo_0007-473x_1967_num_125_3_4868 [consulté le 22/03/2017].

19. - LA SPPEF (Société pour la protection des paysages et de l'esthétique de la France) par exemple, est une des plus anciennes associations de défense du patrimoine. Fondée en 1901, elle a joué un rôle essentiel dans les années 1970 et 1980 dans des combats majeurs contre les atteintes au patrimoine, comme lors de l'affaire du quartier des Halles à Paris. Autre exemple, l'association VMF (Vieilles maisons françaises), créée en 1958, régulièrement représentée dans les commissions régionales et nationales et très présente auprès des propriétaires.

20. - Le conseil des ministres du 2 août 1978 déclare l'année 1980 « Année du Patrimoine ». L'initiative s'est traduite par de nombreuses expositions, manifestations, portes ouvertes destinées au grand public. Elle a contribué à diffuser l'idée de patrimoine dans la société française.

21. - Décret $n^{\circ}$ 84-1007 du 15 novembre 1984 instituant auprès des commissaires de la République de région une commission régionale du patrimoine historique, archéologique et ethnologique. Chaque COREPHAE comprend 30 membres désignés par le commissaire de la République de région : 10 fonctionnaires de l'État, 16 personnalités qualifiées dans le domaine du patrimoine dont 8 titulaires d'un mandat électif national ou local, 4 représentants d'associations ayant pour objet de favoriser la connaissance, la protection et la conservation du patrimoine.

22. - Décret nº 99-78 du 5 février 1999.

23. - Voir sur ce sujet SIRE, Marie-Anne. La France du patrimoine : les choix de la mémoire. Paris : Gallimard/Éditions du patrimoine, 1996 ; CHOAY, Françoise. L’Allégorie du patrimoine. Paris : Seuil, 1999 ; BERCÉ, Françoise. Des monuments historiques au patrimoine. Paris : Flammarion, 2000.

24. - Voir dans la base Mérimée : notice PA00088720.

25. - AMIEL, Christiane, FRANÇOIS, Michèle, BARRÈS, Renaud. Le village des Carrats à Port Leucate. Une utopie sociale et architecturale. Sigean : Parc naturel régional de la Narbonnaise, coll. « Les Carnets du Parc », 2016. 
26. - L'ouverture de la procédure résulte soit d'une demande d'un pétitionnaire (propriétaire, association patrimoniale, collectivité territoriale, etc.) soit d'une initiative des services de l'État (articles R621-2 et R621-53 du Code du patrimoine). L'instruction administrative proprement dite commence dès réception de la demande par un accusé de réception envoyé au pétitionnaire.

27. - La délégation permanente de la CRPS correspond à l'institutionnalisation du groupe préparatoire à la COREPHAE dont la mise en place avait été recommandée par circulaire du 26 septembre 1990. La compétence consultative de la délégation permanente est strictement encadrée par l'article R612-2 du Code du patrimoine : la délégation ne peut émettre, au nom de la CRPS, qu'un avis défavorable lorsqu'elle constate que l'intérêt de l'immeuble proposé est manifestement insuffisant. Dans tous les autres cas, la délégation permanente ne peut que renvoyer l'examen du dossier à la formation plénière de la CRPS, seule compétente pour émettre un avis favorable sur la proposition ou la demande de classement ou d'inscription (voir circulaire du 8 février 2013 relative à la commission régionale du patrimoine et des sites et à l'instruction de certaines autorisations de travaux et décret $n^{\circ} 2006-672$ du 8 juin 2006).

28. - Circulaire du 8 février 2013. Le rapport consiste en un exposé synthétique comprenant : - une présentation de la documentation réunie, sous la forme d'une description des principales caractéristiques historiques, architecturales, décoratives ou techniques de l'immeuble ; - une information sur le déroulement de la procédure, la situation de propriété, les règles d'urbanisme applicables, l'existence d'autres protections ou de réglementations applicables à l'immeuble, à son environnement et aux objets mobiliers qu'il contient, l'ouverture au public, l'existence ou la demande d'une convention d'exonération des droits de mutation, la position du propriétaire relativement à la protection envisagée et ses intentions, éventuellement celle de la collectivité, les négociations en cours, les projets ou menaces concernant l'immeuble, etc. - une information sur les conséquences juridiques de la servitude générée par la mesure de protection : évaluation sommaire, notamment au regard de l'enjeu patrimonial et de la charge de travail créée pour les services gestionnaires. »

29. - Circulaire du 8 février 2013 : « - l'avis du conservateur régional des Monuments historiques, s'appuyant sur les agents du service et notamment le conservateur des $\mathrm{MH}$ : il porte sur l'intérêt historique, artistique, architectural et technique de l'immeuble et de ses décors et d'autre part sur la capacité du bien à être conservé ; - l'avis de l'architecte des bâtiments de France sur l'intérêt architectural de l'immeuble et son état sanitaire ; cet avis pourra être assorti d'une proposition de périmètre de protection adapté ; - un avis peut être également demandé à l'architecte en chef des monuments historiques sur les mêmes bases que le précédent ; - l'avis du service de la région chargé de l'Inventaire général du patrimoine qui replace l'édifice dans un corpus régional ou (et) national ; cet avis portera notamment sur la singularité, la représentativité ou l'exemplarité de l'immeuble."

30. - Extrait de l'article L621-25 du Code du patrimoine.

31. - En aval des commissions, le CPMH est notamment requis pour la rédaction des procès-verbaux des séances.

32. - JAMOT, Francis, MARX, Jean, AUDIBERT, Martine, DENANTE, Sylvie. La protection des immeubles au titre des monuments historiques : manuel méthodologique. Paris : ministère de la Culture et de la Communication, direction de l'Architecture et du Patrimoine, 2003. 
33. - Le cimetière allemand de Veslud (Aisne), par exemple, inscrit au titre des monuments historiques en 1999 ; voir : http://www.culture.gouv.fr/public/mistral/ merimee_fr?ACTION=CHERCHER\&FIELD_1=INSEE\&VALUE_1=02791.

34. - Art. R621-8 du Code du patrimoine.

35. - En application de l'art. R621-58 du Code du patrimoine.

36. - Art. L126 du Code de l'urbanisme.

37. - Voir le dossier : http://www.culture.gouv.fr/public/mistral/merimee_fr?

ACTION=RETROUVER\&FIELD_1=INSEE\&VALUE_1=71332\&NUMBER $=1 \& G R P=0 \& R E Q=\% 28 \%$

$2871332 \% 29 \% 20 \% 3$ aINSEE\% $20 \% 29 \&$ USRNAME=nobody\&USRPWD $=4 \% 24 \%$

$2534 \mathrm{P} \& S P E C=9 \& S Y N=1 \& I M L Y=\& M A X 1=1 \& M A X 2=100 \& M A X 3=100 \& D O M=$ Tous.

38. - Arrêté ministériel du $1^{\mathrm{er}}$ avril 2014 portant classement au titre des monuments historiques de l'église Sainte-Ségolène en totalité.

39. - Construite en 1894 ou 1895, probablement montée à Courbevoie dans la cour de l'atelier du constructeur, on la retrouve de 1896 à 1900 en bord de Seine, où elle est utilisée comme bureau des ingénieurs en charge de la construction du pont Alexandre III. En 1900, elle est remontée dans le bois de Vincennes pour servir de pavillon de l'administration de l'Exposition universelle. Ensuite, elle est rachetée par des maîtres de forges qui installent la maison, alors nommé chalet de fer, dans une propriété de l'Eure, au Plessis, commune de Touffreville, où elle sert de pavillon d'agrément. En 1935, elle est déplacée à Oissel (Seine-Maritime) par le chauffeur mécanicien du maître de forges, qui l'utilise comme garage automobile et logement. Depuis cette date, elle est restée à ce même emplacement.

40. - GALLO, Emmanuelle. «À quoi servent (encore) les historiens de l'architecture ?». Le Moniteur, 11 février 2005.

41. - Sur la complémentarité, parfois difficile, entre les Monuments historiques et l'Inventaire, on se reportera aux pages qui y sont consacrées dans : HEINICH, Nathalie. La fabrique du patrimoine. "De la cathédrale à la petite cuillère ». Paris : Éditions de la Maison des sciences de l'homme, coll. «Ethnologie de la France », 2009.

42. - C'est en 1888 que M. Pic achète au cœur du village un terrain afin d'édifier un bâtiment recyclant l'énergie hydraulique. Sa conception est à mettre en parallèle avec la diffusion des batteuses à vapeur qui s'installaient sur la place des villages. La batteuse, bâtiment privé, mais d'un usage communautaire pour Audressein et pour les villages environnants, aurait fonctionné jusqu'en 1939, la cessation d'activité étant symboliquement liée à la déclaration de guerre.

43. - JUHEL, Enora, MICHARD, Isabelle. « La protection et la gestion urbaine du site de Bataville». Monumental. Revue scientifique et technique des monuments historiques, « Le patrimoine industriel », 2015, p. 46-49.

44. - Voir le site : http://www.scmha.fr/societe-scmha/historique [consulté le 27/03/2017].

45. - COREPHAE puis CRPS d'Alsace, puis CRPS d'ACAL.

46. - On peut retenir l'exemple de la commune de Villé (Bas-Rhin) où les protections récentes ont permis d'étendre la protection de l'enclos paroissial de Villé qui ne comptait qu'un monument historique jusque-là : le cimetière bourgeois (ISMH 12/07/1995). Cette démarche politique, relayée par des actions en faveur de la valorisation du patrimoine (participation au financement d'un ouvrage de la collection « Itinéraire du patrimoine », organisation de visites guidées lors des Journées européennes du patrimoine, financement de plusieurs supports de visites, etc.), constitue dans l'esprit des élus de cette commune un premier pas susceptible d'encourager des propriétaires privés à 
demander la protection de leur maison. Voir aussi : ALBERTONI, Clémentine, HAEGEL, Olivier, SCHEURER, Marie Philippe. L'ensemble paroissial de Villé (Bas-Rhin). Lyon : Lieux Dits éditions, coll. « Parcours du patrimoine », 2013.

47. - Sous la direction de François Goven et Vincent Guigueno.

48. - Voir le site : http://www.culturecommunication.gouv.fr/Regions/Drac-Bretagne/ Politique-et-actions-culturelles/Protection-et-restauration-des-monuments-historiques/ Protection-des-immeubles-au-titre-des-monuments-historiques/13-phares-monumentshistoriques-en-Bretagne.

49. - Voir le site : http://www.culturecommunication.gouv.fr/Regions/Drac-Bretagne/ Politique-et-actions-culturelles/Protection-et-restauration-des-monuments-historiques/ Protection-des-immeubles-au-titre-des-monuments-historiques/Les-phares-monumentshistoriques-des-cotes-de-Bretagne.

50. - Voir le site : http://www.culturecommunication.gouv.fr/Regions/Drac-Paca/ Politique-et-actions-culturelles/Conservation-regionale-des-monuments-historiques/ Monuments-aux-morts-de-la-guerre-de-1914-1918.

51. - GAVIGNAUD-FONTAINE, Geneviève, VAYSSETTES, Jean-Louis, SAUGUET, Michel. Caves coopératives en Languedoc-Roussillon. Lyon : Lieux Dits éditions, 2010. Voir le site : http://patrimoine-culturel.caves-cooperatives.fr/ [consulté le 28/03/2017).

52. - Voir le site : http://www.culture.gouv.fr/culture/inventai/patrimoine/.

53. - « La Cité paysanne de Marckolsheim (1940-1944). Étude et protection d'un quartier de fermes modèles en Alsace ", communication de Clémentine Albertoni, Séverine Wodli et Laurine Sandoval au colloque « La ferme moderne. Architecture rurale et constructions agricoles au $\mathrm{xx}^{\mathrm{e}}$ siècle. Les campagnes européennes et les territoires coloniaux, entre tradition et innovation ", organisé les 11 et 12 octobre 2012 par l'INHA, Paris.

54. - ALBERTONI, Clémentine, SANDOVAL, Laurine et WODLI, Séverine. « La Cité paysanne de Marckolsheim (1940-1944). Étude et protection d'un quartier de fermes modèles en Alsace ", In Situ [En ligne], 21 | 2013, mis en ligne le 22 juillet 2013, consulté le 28 mars 2017. URL : http://insitu.revues.org/10500 ; DOI : 10.4000/insitu.10500.

55. - Association des personnels de documentation des affaires culturelles. Levez le voile sur un métier du patrimoine : recenseur documentaliste chargé de la protection des MH. Octobre 1994.

56. - Éléments du débat de la CRPS : « la rareté d'une telle découverte avait été soulignée et également le fait que c'était un clin d'œil à notre époque qui a interdit ce genre de publicité murale ». « La taille importante du sujet, la clarté de l'inscription, la datation précise et l'état exceptionnel de la couche picturale sont autant de raisons justifiant la nécessité de conserver ces témoignages pratiquement intacts de « réclames » datant de plus d'un siècle. L'inscription au titre des monuments historiques est nécessaire pour reconnaître la qualité de ces peintures et faciliter la restauration à venir ». "Il a été considéré que cette découverte était une opportunité très rare du point de vue de l'histoire de la ville. L'inscription au titre des monuments historiques sera fondamentale pour la reconnaissance de ce témoignage important d'un passé à la fois proche et lointain ». Voir le site : http://www.culture.gouv.fr/public/mistral/dapamer_fr. 57. - Le centre commercial d'Épernay a été rasé, ceux de Ris-Orangis et Reims-Tinqueux dénaturés.

58. - Voir le site : http://www.culture.gouv.fr/public/mistral/merimee_fr.

59. - Label « Patrimoine du xx ${ }^{\mathrm{e}}$ siècle », label « Jardin remarquable », label « Maison des illustres », label « Villes et pays d'art et d'histoire ». 
60. - Y compris la gestion administrative des labels avec l'organisation des commissions d'attribution et les courriers y afférents.

61. - La protection du patrimoine architectural du $\mathrm{xx}^{\mathrm{e}}$ siècle a fait l'objet d'une recommandation du Conseil de l'Europe dès 1991 ( $n^{\circ} \mathrm{R}(91) 13 \mathrm{du}$ Conseil de l'Europe (9 septembre 1991), laquelle, « observant que l'architecture du vingtième siècle fait partie intégrante du patrimoine historique de l'Europe, et que la sauvegarde et la mise en valeur de ses éléments les plus significatifs répondent aux mêmes objectifs et aux mêmes principes que ceux de la conservation du patrimoine architectural dans son ensemble; et constatant que cette catégorie s'avère, en raison de sa proximité dans l'Histoire, de l'abondance de ses témoignages et de son caractère hétérogène, moins reconnue de la part des autorités responsables et du public que d'autres composantes du patrimoine architectural [...], a recommandé de mettre en œuvre [...] des stratégies d'identification, d'étude, de protection, de conservation, de restauration et de sensibilisation ». Cette recommandation s'est traduite en droit français par l'instauration du label « Patrimoine du XX ${ }^{\mathrm{e}}$ siècle » précisé dans les circulaires du 18 juin 1999 et du $1^{\mathrm{er}}$ mars 2001.

62. - Voir le site : http://atlas.patrimoines.culture.fr/atlas/trunk/.

63. - Voir le site : https://www.geoportail.gouv.fr/ [consulté le 28/03/2017].

64. - Voir le site : http://www.culture.gouv.fr/public/mistral/merimee_fr?

ACTION=RETROUVER\&FIELD_1=cmer1\&VALUE_1=\%27LANGUEDOC-ROUSSILLON\%

27\&FIELD_2=cmer4\&VALUE_2=\&FIELD_3=cmer5\&VALUE_3=\&FIELD_4=AUTR\&VALUE_4=\&FIELD_5=TOUT\&VALUE_5=\&FIELD_ $\% 20$ courant\&VALUE_6=\&FIELD_7=date\%

20protection\&VALUE_7=\&FIELD_8=DOSURLP\&VALUE_8=\%20\&NUMBER=50\&GRP=5\&REQ=

\%28\%28\%27LANGUEDOC-ROUSSILLON\%27\%29\%20\%3aLOCA\%2CPLOC\%2CINSEE\%20\%

29\&USRNAME $=$ nobody\&USRPWD $=4 \% 24 \%$

2534P\&SPEC=9\&SYN=1\&IMLY=\&MAX1=1\&MAX2=100\&MAX3=100\&DOM=MH.

65. - Ministère de la Culture et de la Communication, direction générale des Patrimoines, service du Patrimoine. Bilan annuel de la protection des monuments historiques, 2014.

\section{RÉSUMÉS}

Depuis l'époque de Ludovic Vitet et Mérimée, l'État a considérablement développé, étendu et précisé sa politique de préservation du patrimoine bâti. Aux premiers érudits chargés de repérer et documenter les édifices ont succédé les recenseurs des Monuments historiques. L'association MoHiCan, fondée en avril 1960, réunit ces professionnels qui sont aujourd'hui amenés à s'interroger sur les outils et leurs missions en prenant en compte l'évolution de la notion de patrimoine et du sens que la société donne à celle-ci. Cet article revient sur les fondements du métier de « délégué chargé du recensement des monuments historiques et des sites à la direction de l'Architecture du ministère d'état aux Affaires culturelles " pour les comparer à ses activités actuelles. Interlocuteur permanent des propriétaires, des partenaires publics et privés, du monde universitaire et associatif, des autres services de l'État ou des collectivités, cet acteur incontournable de la chaîne patrimoniale est également le médiateur d'un patrimoine régional qu'il connaît bien. Le dynamisme de l'association de ces professionnels, MoHiCan, en témoigne. À l'aide d'exemples précis, cet article démontre en quoi leur intervention sur le terrain et leurs 
recherches sont déterminantes pour la reconnaissance patrimoniale d'un édifice, qui peut aller parfois jusqu'à l'élaboration d'une stratégie juridique de sa défense. Enfin, à travers leurs compétences scientifiques et administratives de chargés de la valorisation, d'animateurs de réseau ou encore de gestionnaires de bases de données, on reconnaîtra la polyvalence et la technicité de leurs pratiques documentaires, qui déterminent leur place incontournable dans la procédure de protection au titre des monuments historiques.

Since the beginnings of historic monuments protection in France during the 1830s, inspired by figures such as Ludovic Vitet and Prosper Mérimée, the State has considerably developed and refined its policies for the preservation of the built historic environment. To begin with the identification and documentation of buildings worthy of protection was carried out by local amateurs and scholars. Official historic monuments 'recenseurs' (surveyors, inspectors or enumerators) then took over this task. MoHiCan is an association founded in April 1960 to bring these professional heritage surveyors together. It aims to help them reflect collectively about the contemporary questions concerning the tools available for their missions and the nature of these missions at a time when the very notion of heritage, and the meaning given to this notion by society, is witnessing considerable change. This article goes back to the original definition of the rôle of these 'recenseurs', as 'delegates entrusted with the survey of historic monuments and sites at the architectural directorate of the ministry of cultural affairs', comparing this original mission with the present-day activities of these heritage professionals. The recenseur is a vital actor in the process of defining heritage, in permanent contact with monument owners and with other official services at State and local levels. He or she is also someone who, thanks to his or her knowledge of the field, can interpret a region's heritage. The dynamism of the MoHiCan association bears witness to the vitality of the ways these different missions are carried out. Using precise examples, the article will show how field research and documentary enquiries in libraries and archive depots play a decisive part in how a building comes to be recognised for its heritage values. This recognition can also involve the definition of legal strategies to ensure the preservation of the monument. Thanks to their scientific and administrative expertise in the tasks of interpreting the heritage, of animating networks or managing data bases, the variety of their skills and the technical seriousness of their documentary work, these recenseurs occupy an essential and central rôle in the procedures of historic monuments preservation.

\section{INDEX}

Mots-clés : patrimoine, ministère de la culture, protection des monuments historiques, inventaire, recenseur, chargé de la protection des monuments historiques, architecture, code du patrimoine

Keywords : heritage, ministry of culture, historic monuments designation, inventory, 'recenseur', surveyor, architecture, heritage legislation

\section{AUTEURS}

ENORA JUHEL

Chargée de la protection des monuments historiques, DRAC Grand Est (Metz)

enora.juhel@culture.gouv.fr 


\section{SOPHIE LOPPINET-MÉO}

Chargée de la protection des monuments historiques, DRAC Bourgogne-Franche-Comté (Dijon) sophie.loppinet@culture.gouv.fr

\section{CLÉMENTINE ALBERTONI}

Chargée de la protection des monuments historiques, DRAC Grand Est (Strasbourg)

clementine.albertoni@culture.gouv.fr 